\title{
Le Long dimanche de fiançailles de Mathilde Donnay. De la féminité du récit narratif à la féminité du récit filmique : deux femmes pour un destin
}

\author{
José Luis Arráez \\ Universidad de Alicante \\ jl.arraez@ua.es
}

Rebut: 28 novembre de 2014

Acceptat: 18 de febrer de 2015

\section{RESUM}

El larg diumenge de festeig de Mathilde Donnay. De la feminitat del relat narratiu a la feminitat del relat fílmic: dues dones per a un destí

El principal objectiu de la nostra investigació és l'anàlisi comparativa de la personalitat de Mathilde (r), l'herö̈na de la novel-la Un long dimanche de fiançailles escrita per Sébastien Japrisot, i de Mathilde (f), l'heroïna de la pel-lícula homònima el guió de la qual ha sigut escrit per Jean-Pierre Jeunet i Guillaume Laurant. El nostre estudi permetrà desvetllar les respectives particularitats de la feminitat de les dues « veuves blanches » després de la I Guerra mundial, i gràcies al salt enrere de la narradora a les respectives feminitats abans que la guerra haguera esgarrat els seus festejos. Com a instruments d'aprofundiment en els discursos literari i fílmic hem utilitzat la narratologia literària i fílmica. D'altra banda, hem introduït el model semiològic, ja que per a accedir a la configuració de la feminitat de Mathilde (f) hem centrat la nostra atenció en els signes visibles en la pantalla. Finalment, hem recorregut a les interpretacions simbòliques, ja que açò ens permetrà accedir a les esferes de les idees de Mathilde (f) i Mathilde (r).

Paraules Clau

Mathilde, feminitat, simbolisme, guerra, film, narració. 


\section{RÉSUMÉ}

Le Long dimanche de fiançailles de Mathilde Donnay. De la féminité du récit narratif à la féminité du récit filmique : deux femmes pour un destin Au cours de cet article, nous envisageons l'analyse comparative de la personnalité de Mathilde(r), l'héroïne du roman Un long dimanche de fiançailles de Sébastien Japrisot, et de Mathilde(f), l'héroïne du film éponyme dont le scénario appartient à Jean-Pierre Jeunet et Guillaume Laurant. Notre étude permettra de dévoiler les particularités de la féminité des deux « veuves blanches » au lendemain de la Grande Guerre, et moyennant les flashbacks de la narratrice, de leur féminité avant que la guerre ne déchire pas leurs fiançailles. À cette fin, nous avons utilisé la narratologie littéraire et filmique comme instruments d'approfondissement dans les discours littéraire et filmique. D'autre part, nous avons introduit le modèle sémiologique étant donné que pour arriver à la configuration de la féminité de Mathilde(f), nous devons porter notre attention sur le système de signes visibles sur l'écran. Nous avons également fait appel aux interprétations symboliques afin d'accéder au plan des idées de Mathilde(f) et de Mathilde(r).

\section{Mots Clé}

Mathilde, féminité, symbolisme, guerre, film, narration.

\section{RESUMEN}

El Largo domingo de noviazgo de Mathilde Donnay. De la feminidad del relato narrativo a la feminidad del relato fílmico: dos mujeres para un destino

El principal objetivo de esta investigación es el análisis comparativo de la personalidad de Mathilde(r), la heroína de la novela Un long dimanche de fiançailles escrita por Sébastien Japrisot, y de Mathilde(f), la heroína de la película homónima cuyo guión pertenece a Jean-Pierre Jeunet y Guillaume Laurant. Nuestro estudio permitirá desvelar las particularidades de la feminidad de las dos « veuves blanches » tras la I Guerra mundial, y gracias a los flashbacks de la narradora de sus respectivas feminidades antes de que la guerra desgarrase sus noviazgos. Como instrumentos de profundización en los discursos literario y fílmico hemos utilizado la narratología literaria y fílmica. Por otro lado, hemos introducido el modelo semiológico puespara acceder a la configuración de la feminidad de Mathilde(f) hemos centrado nuestra atención en lo signos visibles en la pantalla. Por último, hemos recurrido a las interpretaciones simbólicas, puesto que ello nos permitirá acceder a las esfera de la ideas de Mathilde(f) y Mathilde(r).

Palabras Clave

Mathilde, feminidad, simbolismo, guerra, film, narración. 


\begin{abstract}
The A Very Long Engagement of Mathilde Donnay. Of feminite narrative account of the feminite of filmic story: two women to a destination

Our main concern in this research is the comparative analysis of Mathilde's personality, the heroine of the novel Un long dimanche de fiançailles written by Sébastien Japrisot, and that of Mathilde, the heroine of the homonymous picture whose script has been written down by Jean-Pierre Jeunet and Guillaume Laurant. Our study will allow us to unveil the respective particularities about the feminity of both «veuves blanches» after the I World War, and thanks to the narrator's flashbacks, their respective feminities before the War would have torn their engagements. As an instrument to go in depth in the literary and film discourses we have used the literary and film narratology. On the other hand, we have introduced a semiological pattern, because we have put all our attention in the screen visible signs in order to gain acces to Mathilde's feminity configuration. Lastly, we have appealed to symbolic interpretations, in the conviction that they will allow us to get to Mathilde's(f) and Mathilde's(r) ideas sphere.
\end{abstract}

KEYWORDS

Mathilde, feminite, semiological, war, film, narration.

\title{
Préliminaires
}

Pour les femmes à qui la Grande Guerre avait arraché un être cher et empêché d'enterrer ses dépouilles mortelles, le deuil fut éternel. La réception d'un document officiel du ministère de la Défense officialisant le décès ne calmait ni leur douleur ni leur désespoir. Louise Ménard-Lemeunier, veuve d'un combattant disparu au front, témoignera de la douleur causée par l'absence de la dépouille de son mari dans les derniers vers de Jour de Toussaint :

Tous n'ont pas, cependant, pour ceux qu'ils ont perdus,

À l'ombre d'un clocher, une tombe en partage.

Combien parmi nos morts ne sont pas revenus,

Dans leur pays natal, reposer au village!

Les parents de ceux-là restent seuls au foyer. 
Qu'iraient-ils faire ainsi dans une nécropole?

Leurs regards embués n'ont pas où se poser,

Car leur esprit, au loin, bien tristement s'envole ${ }^{1}$

Certaines d'entre ces veuves, qu'elles fussent mères, épouses ou fiancées acceptèrent avec résignation cette perte ; par contre, nombre d'autres gardèrent à l'espérance de les retrouver en vie et ne cessèrent de chercher, d'interroger et de consulter dans les limites du possible, même de l'impossible, dans l'intention de découvrir un contact ou une information encourageant leurs recherches. Même après la signature des traités de paix, des centaines de femmes conservèrent l'espoir de voir rentrer leurs proches officiellement disparus au front.

Certes, la France était victorieuse ; mais la victoire avait un prix très lourd : des milliers de femmes et d'hommes déchirés par la mort d'un proche. Le pays en cours de reconstruction inventait un nouveau statut civil pour les jeunes femmes dont les fiancés mobilisés étaient tombés au front, celui des « veuves blanches ». Les historiens S. Audoin-Rouzeau et A. Becker y relèveront l'introduction de cette expression : « La guerre a $\mathrm{vu}$, il est vrai, l'apparition d'un terme pour désigner les fiancées de ceux qui avaient été tués trop tôt pour qu'elles aient pu devenir des épouses $»^{2}$.

Les histoires réelles de ces fiancées fidèles à leur promesse d'avantguerre ont été mises, par la suite, au service de la littérature ou du cinéma, dans des histoires fictives.

La littérature s'est particulièrement emparée de la quête poignante menée par certaines de ces «veuves blanches». Ainsi, certains titres comme Les Veuves blanches (1926) de Marcel Priollet ou Un long dimanche de fiançailles de Sébastien Japrisot ${ }^{3}$ se situent en France au lendemain du conflit.

\footnotetext{
${ }^{1}$ Louise MENARD-LEMEUNIER, « Jour de Toussaint », in Le Trait d'union, novembre 1924, Archives Départementales de Maine-et-Loire, 126 JO 1. Le trait d'union est un organe de la Fédération des victimes de la guerre.

2 Stéphane AUDOIN-ROUZEAU et Annette BECKER, 14-18, retrouver la Guerre, Paris, Gallimard, 2000, p. 201.

${ }^{3}$ Un long dimanche de fiançailles, Paris, Denoël, 1991. Prix Interallié 1991.

Par la suite, les citations tirées de ce roman sont indiquées dans le texte avec le numéro de la page précédé des abréviations suivantes entre parenthèses $(L D F$, ) suivies des numéros de page renvoyant à l'édition citée supra.
} 


\section{Introduction}

En 1991, le jury du Prix Interallié décernait son prix annuel au roman Un long dimanche de fiançailles de Sébastien Japrisot. Écrivain éclectique, auteur de plusieurs romans, récits et scénarios, la célébrité de Japrisot lui était surtout due à ses romans policiers et à leur adaptation cinématographique. Bientôt devenu un phénomène littéraire, ce roman lauréat sera catalogué par certains critiques, tels Loïc Artiaga ${ }^{4}$, Paul Bleton ${ }^{5}$ ou Pierre Schoentjes ${ }^{6}$, parmi les romans populaires en raison de ses personnages, des motifs et des péripéties de son intrigue. C'est sans doute son hétérogénéité générique qui a contribué à son grand succès éditorial : en effet, sous un titre sentimental et intriguant, le romancier a combiné simultanément un roman historique, un roman à énigme, un roman sentimental et un roman psychologique.

En 2004, l'adaptation de ce roman-polyèdre par Jean-Pierre Jeunet et Guillaume Laurant est projetée sur grand écran, accueillie à la presque unanimité par les acclamations du public, les éloges de la critique, et de nombreuses distinctions dans certains des plus importants festivals cinématographiques internationaux ${ }^{7}$.

${ }^{4}$ Loïc ARTIAGA, Le roman populaire : des premiers feuilletons aux adaptions télévisuelles, 1836-1960, Paris, Autrement, 2008, p. 152.

${ }^{5}$ Paul BLETON, «Les fortunes médiatiques du roman populaire », in ibid., pp. 137- 155.

${ }^{6}$ Pierre SCHOENTJES (éd.), La Grande Guerre : Un siècle de fictions romanesques, Genève, Librairie Droz, 2008, p. 273.

${ }^{7}$ Palmarès et nominations :

- César 2005 de la meilleure actrice dans un second rôle (Marion Cotillard)

- César 2005 du meilleur espoir masculin (Gaspard Ulliel)

- César 2005 de la meilleure photographie (Bruno Delbonnel)

- César 2005 des meilleurs costumes (Madeline Fontaine)

- César 2005 du meilleur décor (Aline Bonetto)

- Nommé César du Meilleur film français de l'année

- Nommé César du Meilleur réalisateur Jean-Pierre Jeunet

- Nommé César de la Meilleure actrice Audrey Tautou

- Nommé César du Meilleur scénario original ou adaptation Jean-Pierre Jeunet, Guillaume Laurant

- Nommé César de la Meilleure musique écrite pour un film Angelo Badalamenti

- Nommé César du Meilleur son Gérard Hardy, Vincent Arnardi, Jean Umansky

- Nommé César du Meilleur montage Hervé Schneid

- Dallas-Fort Worth Film Critics Association : Meilleur film en langue étrangère 2005

- Florida Film Critics Circle : Meilleur film étranger 2005

- Chicago Film Critics Association : Meilleur film en langue étrangère 2005

- Festival international du Making-of : Grand prix du Jury 2005

- Prix Edgar-Allan-Poe 2005 du meilleur scénario

- Nommé Oscar 2005 de la meilleure photographie (Bruno Delbonnel) 
En s'appuyant sur les critiques littéraires et cinématographiques, on montrera que ce qui dans le roman avait charmé les lecteurs et dans le film séduit les spectateurs, c'était le personnage de Mathilde inventé par Japrisot, réinventé par Jeunet et Laurant et interprétée par Audrey Tautou, l'éternelle Amélie Poulain.

Mathilde Donnay, l'héroïne des textes narratif et filmique, se dédouble en deux personnages différents, selon qu'on réalise l'analyse du récit narratif ou celle du récit filmique. L'histoire est cependant la même, celle de Mathilde, «veuve blanche » au lendemain de la Grande Guerre, à la recherche de son fiancé Manech que, intuitivement, elle croit vivant, bien que, officiellement, il soit considéré comme décédé après «sa condamnation à mort pour l'exemple » par un conseil de guerre, à la suite d'une mutilation volontaire. Cette première recherche en entraîne une seconde, à savoir, la réalité des circonstances qui entourent, sur les lieux de la fusillade, l'abandon de Manech, les mains liées, sur le no man's land, à la merci des coups de fusil tirés par les soldats allemands depuis leurs tranchées toutes proches ${ }^{8}$.

Bien que lui faisant vivre la même histoire d'amour, d'attente et de recherche, l'imagination et la créativité de leurs auteurs dotent chaque Mathilde d'une personnalité différente. Nous avouerons même, que la Mathilde

- Nommé Oscar 2005 des meilleurs décors (Aline Bonetto)

- Festival international de Hong Kong - 2005

- Prix Les Lumières - 2005

- Prix du Festival Français de Moscou - 2004

- Prix Festival long-métrage Russie

- Festival international du film de Stockholm - Section «World Cinema » - 2004

- Nommé Golden Globes 2005 - Meilleur film en langue étrangère

- Nommé BAFTA Awards / Orange British Academy Film Awards 2005 - Meilleur film non anglophone

- Nommé NRJ Ciné Awards 2005 - meilleure bande-annonce

- Nommé NRJ Ciné Awards 2005 et meilleure actrice Marion Cotillard

${ }^{8}$ Il y a une abondante littérature et filmographie concernant les exécutions « pour l'exemple ». Philippe Baudorre nous offre une liste très intéressante : La Der des ders de Didier Daeninckx (1985), Le Boucher des Hurlus de Jean Arnila (1982), The Paths 01 Glory de Stanley Kubrick (Etats-Unis, 1957), projection autorisée en France en 1975 sous le titre Les Sentiers de la gloire), Joseph Losey, King and country, Pour l'exemple (1964), ou Francesco Rosi, Uomini Contro, Les Hommes contre (1970), Le Valet de gloire de Joseph Jolinon (1923), Pain de soldat d'Henri Poulaille (1937), Clavel soldat de Léon Werth (1919), Louis Guilloux, Le Sang noir (1935), Les Croix de bois de Roland Dorgelès (1919), Feu d'Henri Barbusse (1916).

Cf. Philippe BAUDORRE, " Pour l'exemple », in Richard JACQUEMOND (dir), Histoire et fiction dans les littératures modernes. (France, Europe, monde arabe). L'écriture de l'histoire. Volume 2, Paris, L'Harmattan, 2006, pp. 29-44. 
filmique l'emporte sur la Mathilde romanesque ${ }^{9}$. Cette supériorité revient de toute évidence à la direction de Jean-Pierre Jeunet. Le scénariste et critique cinématographique Philippe Piazzo partage cette préférence :

Un long dimanche de fiançailles, le film est, comme son modèle, un vrai feuilleton à suspense, mais qui est aussi complètement remodelé par l'univers visuel de Jean-Pierre Jeunet, pour qui un film semble être [...] toujours créateur de surprises. Il n'y a donc pas un, mais dix films, ici. Et le miracle, c'est que tous sont réussis ${ }^{10}$.

Nous envisagerons une étude comparative de la personnalité des deux Mathilde prenant la narratologie littéraire et la narratologie filmique comme instruments d'analyse des discours littéraire et filmique. Étant donné que pour définir la configuration de la féminité de Mathilde dans le film, nous devons porter toute notre attention sur le système de signes visibles sur l'écran, nous avons utilisé le modèle sémiologique tel que le présente Georges Mounin : l' «étude de tous les systèmes de signes autres que les langues naturelles $»^{11}$. Nous avons également fait appel aux interprétations symboliques, car, conformément à Todorov, nous estimons que « loin de caractériser la raison abstraite, le symbole est propre à la manière intuitive et sensitive d'appréhender les choses $»^{12}$, c'est ainsi que nous accéderons au plan des idées de Mathilde.

Notre analyse nous permettra de révéler et de réfléchir sur les particularités de la personnalité de chaque « veuve blanche » dans leur «long dimanche de fiançailles », sachant que le dévoilement de leur personnalité nous mènera vers le dévoilement de leur féminité.

\section{Approche à la féminité}

Les innombrables études réalisées dans les différents champs de la connaissance (psychologie, psychiatrie, anthropologie, biologie...) offrent actuellement de multiples théories définissant et déterminant la féminité et la

\footnotetext{
${ }^{9}$ Par la suite, afin de faire la différence entre l'hérö̈ne de roman et l'hérö̈ne du film on emploiera la nomenclature suivante : Mathilde(r) et Mathilde (f).

${ }_{10}$ Philippe PIAZZO in http ://www.allocine.fr/critique/fichepresse_gen_cpresse=82001.html? page $=5$ [page consultée le 20 octobre 2014].

${ }^{11}$ Georges MOUNIN, Introduction à la sémiologie, Paris, Minuit, 1970, p. 68.

${ }^{12}$ Tzevetan TODOROV, Théories du symbole, Paris, Seuil, p. 236.
} 
masculinité. Parmi elles, citons la théorie des stéréotypes soutenant que chacun d'entre eux comprend certains traits de la personnalité, de la conduite, des occupations ou des traits physiques que l'on considère comme caractéristiques des hommes ou des femmes du simple fait de l'être. Les stéréotypes obéissent à des croyances sociales selon lesquelles il est légitime d'attribuer des caractéristiques aux individus en raison de leur sexe.

Notre point de vue s'éloigne de ces théories, car nous ne considérons pas le sexe comme un élément distinctif de la féminité ou de la masculinité. Le but de notre travail est l'exploration de la féminité de Mathilde, mais l'instrument nous permettant d'y accéder ne sera donc pas l'analyse de cette féminité en fonction de son sexe.

Notre point de départ, c'est la conception de la personnalité énoncée par Raymond Cattell pour qui celle-ci « est concernée par tout le comportement de l'individu, par ce qui est manifeste et par ce qui est sous la peau $»^{13}$. À partir de cette doctrine, nous estimons que celles qui se manifestent dans le roman et dans le film, celles qui habitent les personnages sont deux personnes, et non pas deux individus sexués. C'est ainsi que, partant de cette définition, et à travers la conduite en public et en privé des deux Mathilde, nous saisirons leur personnalité, ce qui nous conduira d'autre part à configurer leur féminité.

À la fin du conflit, les bilans officiels recensèrent 600000 veuves de guerre françaises. Logiquement, il serait impossible de dénombrer et de dénommer celles qui furent classées comme des "veuves blanches ». Néanmoins, ces jeunes filles, contraintes au souvenir et au célibat, comme Delphine Arène ${ }^{14}$ ou Jeanne Malvoisin ${ }^{15}$, traversèrent l'histoire de la France et inspirèrent la littérature, le cinéma ou la chanson ${ }^{16}$. Mathilde aurait pu être une de ces nombreuses jeunes françaises célibataires, veuves et endeuillées. Elle est sans doute issue du réel, de la France au lendemain de la Grande Guerre et pendant les « années folles ». Pourtant, dans le cas particulier de Mathilde, on constate une évolution face au référent réel, car elle décide de sortir du noyau

\footnotetext{
${ }^{13}$ Raymond B. CATTEL, Personality : A systematic theoretical and factual study, New York, McGraw-Hill, 1950. Cité par Winfrid HUBER, Introduction à la psychologie de la personnalité, Liège, Mardaga, 1977, p. 12.

${ }^{14}$ Voir : http ://www.latribunerepublicaine.fr/Actualite/Bellegarde/2011/08/24/article_delphine_ arene_la_dame_duhaut_bugey.shtml [page consultée le 5 novembre 2014].

15 Jacqueline de Romilly est l'auteur de Jeanne (Paris, Editions de Fallois, 2011), une autobiographie où elle fait un hommage à sa mère, Jeanne Malvoisin, veuve de la Première Guerre mondiale.

${ }^{16}$ Voir «Louise» de Gérard Berliner.
} 
familial et du cercle social pour démasquer les mensonges officiels qui cachent la vérité sur la disparition de Manech.

Dans son essai consacré aux victimes de la Grande guerre, Aurélie Brayet accorde une place importante dans la société à cette nouvelle catégorie de veuvage : «Veuves fidèles à leurs promesses, mais aussi jeunes filles, fiancées et amoureuses, ont parfois refusé de vivre sans l'homme aimé, et sans aller jusqu'à le rejoindre, ont orienté leur vie autour de la fidélité $»^{17}$. Cependant, bien qu'issue du réel, Mathilde s'éloigne de la norme établie, c'est pourquoi nous avons introduit supra le terme «évolution », puisque Mathilde, échappant à la résignation et au deuil à vie, entreprend les démarches administratives et privées adéquates, ainsi que les voyages nécessaires, dans la recherche de son fiancé disparu sur le champ de bataille. Sa décision prise, Mathilde doit surmonter des obstacles, parmi lesquels le plus insidieux est l'opposition des siens, qui lui conseillent d'abandonner son enquête et son espoir pour laisser la place à l'arrivée d'un nouveau fiancé. Son attitude rebelle, inscrit Mathilde dans une féminité non traditionnelle, telle que la définit Daniela RoventaFrumusani :

La féminité « traditionnelle » se construit autour du pôle de la soumission, de la passivité, de la réceptivité sexuelle dans le cas des jeunes femmes et de l'attitude protectrice chez les jeunes mâles. En revanche, la féminité «non traditionnelle » se construit comme autonomie et résistance à la culture dominante ${ }^{18}$.

Nous compléterons cette définition par la théorie de Kate Millet pour qui

la personnalité humaine se forme selon des lignes stéréotypées définies par la catégorie sexuelle ( masculin » et «féminin »), fondées sur les besoins et les valeurs du groupe dominant et dictées par ce que ses membres chérissent en eux-mêmes et trouvent commode chez leurs subalternes : agression, intelligence, force, efficacité chez le mâle ; passivité, ignorance, docilité, « vertu », inefficacité chez la femelle ${ }^{19}$.

\footnotetext{
${ }^{17}$ Aurélie BRAYET, Revivre : victimes de guerre de la Grande guerre à Saint-Étienne 1914-1935, Saint-Étienne, Publications de l'Université Saint-Étienne, 2006, p. 126.

${ }_{18}$ Daniela ROVENTA-FRUMUSANI, Concepts fondamentaux pour les études de genre, Paris, Éditions des archives contemporains, coll. « Archives des études de genre », 2009, p. 26.

${ }^{19}$ Kate MILLET, La Politique du mâle, Paris, Stock, coll. « Points actuels », 1971, p. 40.
} 
L'indépendance, le déterminisme et le courage de Mathilde la situent audelà de ces «veuves blanches » décidées uniquement à perpétuer la mémoire de leur fiancé et à endurer une peine d'enfermement à vie à cause de la pression familiale et sociale. L'historienne Stéphanie Petit dans son essai consacré aux veuves de la Grande Guerre signalera cette pression sociale :

En raison de la représentation sociale des sexes, l'épouse est considérée comme marquée par la semence de son mari, devenant ainsi son unique propriété. De ce fait, elle ne peut ensuite appartenir décemment à un autre homme, de surcroît, si le défunt est un héros ${ }^{20}$.

Outre ces considérations sociales et historiques qui sous-tendent la genèse du personnage, il importe également de considérer chez la Mathilde de Japrisot l'empreinte de ses personnages policiers et chez la Mathilde de Jeunet et Laurant l'aura d'Amélie Poulin. En récupérant Mathilde de l'HISTOIRE à travers les fictions romanesque et filmique, le romancier et les scénaristes démystifient les « veuves blanches », bien que, inconsciemment, ils contribuent à la formation d'un nouveau prototype, celui de « Mathilde », la « veuve blanche » obstinée à la recherche de son bien-aimé.

\section{Caractéristiques de la personnalité de Mathilde à partir de son corps}

Selon Hans Eysenck, la personnalité résulte de « l'organisation plus ou moins ferme et durable du caractère, du tempérament, de l'intelligence et du physique d'une personne $»^{21}$. De même que le psychologue britannique, nous estimons que pour comprendre les enjeux de la personnalité de Mathilde, outre ses variantes psychiques, morales et de l'esprit, on doit également tenir compte de son $\operatorname{corps}^{22}$. À partir de cette idée, nous dégagerons certains traits de la personnalité de Mathilde par rapport à son physique, puisque elle est marquée par son handicap.

\footnotetext{
${ }^{20}$ Stéphanie PETIT, Les veuves de la Grande Guerre, d'éternelles endeuillées ?, Paris, Cygne, 2007, p. 132.

${ }^{21}$ Winfrid HUBER, Introduction à la psychologie de la personnalité, Liège, Mardaga, 1992, p. 12.

${ }^{22}$ Bien entendu, en faisant intervenir le corps, on ne prétend pas établir une différence biologique, on fait référence à la physionomie sans que la sexualité intervienne.
} 
Japrisot et Jeunet-Laurant modèlent deux Mathilde ${ }^{23}$ dont le handicap provoque chez les lecteurs et les spectateurs un accès de tendresse et de compassion dès le début des récits. À ce propos, les déclarations du romancier sont très éclairantes :

J'ai voulu Mathilde exemplaire - elle ne serait pas exemplaire si elle pouvait marcher - je lui ai enlevé tout au départ pour qu'elle se montre telle qu'elle est intérieurement, une passionnée qui va jusqu'au bout [...] Le seul défi au malheur, pour Mathilde, c'est la dérision $(L D F, 362)^{24}$.

Cependant, si la plus évidente caractéristique corporelle de Mathilde est son handicap, la différence la plus significative entre les deux personnages est l'invalidité motrice de Mathilde(r) l'obligeant à se déplacer indéfectiblement sur un fauteuil roulant, d'une part, face, de l'autre, à la paralysie partielle de la jambe gauche (f) qui lui permet de marcher, même de courir, sans aucun appui. Jean Jeunet avoue avoir réalisé cette modification dans une finalité esthétique et dans le but de simplifier le filmage du personnage, dans son rythme et son action. De son côté, Guillaume Laurant assure que « dans le roman, on finit par vite oublier son fauteuil. À l'écran, on aurait vu que ça $»^{25}$. En marge de ces considérations, la seule boiterie métamorphose du point de vue esthétique le personnage original, engendrant un nouveau personnage doté d'une personnalité différente.

Malgré son handicap, Mathilde(r) se montre fière d'elle-même, fière de son état physique, car sa force et son énergie lui permettent d'être indépendante, et par conséquent fière de son tempérament puisque son courage et son orgueil lui permettent d'être autonome. Sur ce, la narratrice ${ }^{26}$ affirme :

Déjà, elle était très orgueilleuse, elle s'était arrangée mieux qu'elle pouvait avec elle-même. Elle n'acceptait d'être aidée de personne, sauf pour son bain, dans

\footnotetext{
${ }^{23}$ Afin de ne pas confondre la Mathilde littéraire avec la Mathilde filmique nous introduirons dans le texte la distinction suivante lorsqu'elles prêtent à confusion : Mathilde(r), Mathilde(f). Pour désigner le personnage sans référence à un texte concret, on emploiera simplement son prénom.

${ }^{24}$ Propos de Sébastien Japrisot cités dans le dossier du roman pour l'édition Folioplus, p. 362.

${ }^{25}$ Ibid., p. 362.

${ }^{26}$ À propos de la narratrice, nous signalerons l'identification entre la narratrice et Mathilde. À ce propos Gabriella Körömi soutient que "Mathilde-narratrice, cachée derrière le masque d'un narrateur anonyme, se tient obstinément à cette identité feinte ». $C f$. Gabriella KÖRÖMI, « Narration d'une double recherche dans Un long dimanche de fiançailles de Sébastien Japrisot », Verbum Analecta Neolatina, XII/2, p. 336.
} 
les endroits où l'on va seule. Sans doute s'est-elle trouvée en difficulté plusieurs fois, sans doute s'est-elle fait mal, mais l'expérience prise, elle a toujours été capable de se débrouiller partout avec ses bras et ses mains, pourvu qu'on ait prévu où il faut de quoi s'accrocher $(L D F, 83)$.

La narratrice reviendra une deuxième fois sur la maîtrise acquise par Mathilde(r) sur son corps, sans doute à cause de son handicap : "Mathilde est une contorsionniste-née » $(L D F, 143)$. La comparaison avec une acrobate confère à sa physionomie un certain charme, celui de la souplesse de son corps en mouvement.

Pourtant, son fauteuil roulant, ses bras et ses mains ne lui donneront qu'une autonomie assez limitée dans ses nombreux déplacements à la recherche d'informations sur Manech. Pour cette raison, elle se fait toujours accompagner par Sylvain ${ }^{27}$, et, quoique ceci n'amoindrisse pas son courage, ce soutien montre la limite de son indépendance.

Par contre, la jambe de Mathilde(f) légèrement atrophiée par la poliomyélite ne l'empêche pas de se déplacer complètement seule à Paris plusieurs fois pour louer les services d'un détective privé, pour visiter son avocat ou pour rencontrer les femmes et les fiancés d'autres militaires condamnés. En plus de l'énergie physique et morale qui anime l'une et l'autre Mathilde, Jeunet et Laurant pourvoient Mathilde(f) d'une autonomie presque absolue.

$\mathrm{Du}$ point de vue esthétique, Japrisot ne prête aucune attention au physique de Mathilde(r) se déplaçant en fauteuil roulant; par contre la caméra filme à maintes reprises la démarche boiteuse de Mathilde(f) sous différents angles et différentes distances. Le film nous offre cinq scènes particulièrement significatives focalisées sur le corps boiteux de la jeune fille.

La première scène reproduit la descente de Mathilde(f), courant et claudiquant à travers des champs escarpés pour aller, après l'adieu, à la rencontre de la voiture de Manech (1:08 - 1:09:24) ${ }^{28}$. Les mouvements de la caméra filment sa course frénétique à travers plusieurs échelles de plans qui oscillent entre le plan de demi-ensemble et le plan moyen. Les gros plans focalisent alternativement en peu de photogrammes ses jambes et son visage, c'est-à-dire, sa souffrance physique et morale. L'effort physique réalisé par la jeune fille se reflète sur son visage crispé et son regard fixe. Jeunet parvient ainsi à transmettre aux spectateurs la volonté et la ténacité de Mathilde(f), pour

\footnotetext{
${ }^{27}$ Sylvain et sa femme Bénédicte sont au service des Donnay depuis l'enfance de Mathilde.

${ }^{28}$ Mathilde a l'habitude de soumettre l'avenir à des suppositions. Dans ce cas-ci, après ses adieux à Manech aux portes de la maison et son départ en voiture, elle suppose que si elle arrive en courant au virage avant que la voiture de Manech, celui-ci reviendra sain et sauf du front.
} 
qui la poliomyélite et un terrain escarpé ne sauraient représenter en aucun cas un obstacle. À plusieurs reprises nous indiquerons l'expressivité faciale de Mathilde(f), selon Léon Deneb « la cara es el lienzo en el que se imprimen, si no los pensamientos, sí los sentimientos $»^{29}$; en ce sens, l'expressivité d'Audrey Tautou permet aux spectateurs de saisir les pensées et les sentiments de la jeune femme.
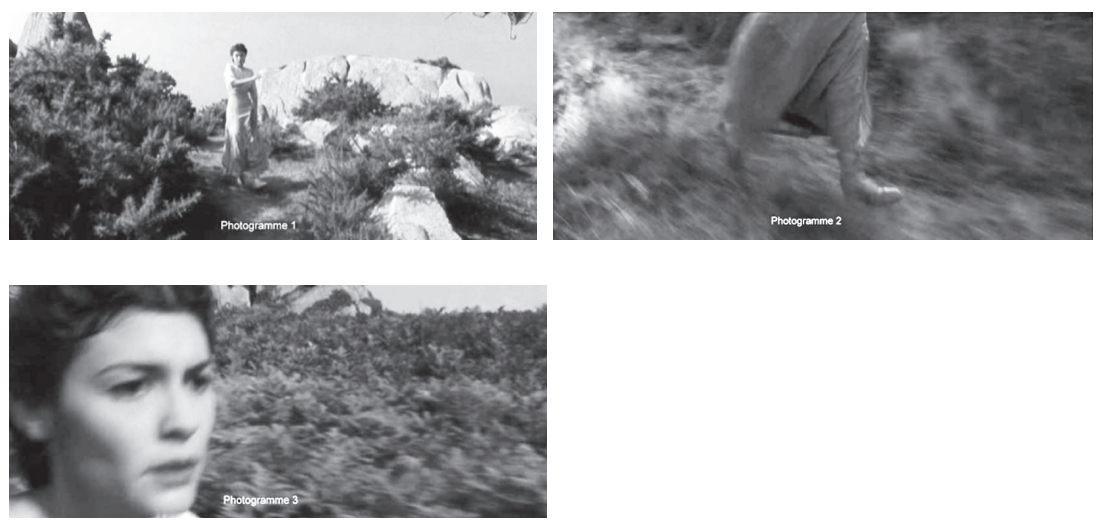

Une deuxième séquence, également révélatrice de sa résistance, de sa ténacité, mais aussi de sa fidélité au souvenir de son fiancé, reproduit la montée de Mathilde(f) jusqu'à la lanterne du phare à travers un escalier à colimaçons pour admirer le crépuscule (12:10 - 12:32). Le déplacement circulaire de la caméra en position de contre-plongée filme son ascension marche après marche, son corps courbé par l'effort, sa main cramponnée fortement à la rampe et sa tête levée vers le haut. Cette scène, métaphore de sa recherche décourageante, témoigne à nouveau de son courage et de son obstination.

Une troisième scène insiste sur la démarche heurtée et saccadée de Mathilde(f) lorsque, à la recherche d'Élodie Gordes ${ }^{30}$, elle sillonne les Halles de Paris $(51: 46$ - 53 :46). À travers un travelling avant et un plan italien

${ }^{29}$ Léon DENEB, Diccionario de símbolos: selección temática de los símbolos más universales, Madrid, Biblioteca nueva, 2000, p. 199.

${ }^{30}$ La femme de Benjamin Gordes, ami et camarade au front de Kléber Bouquet, un des cinq condamnés à mort pour mutilation volontaire. 


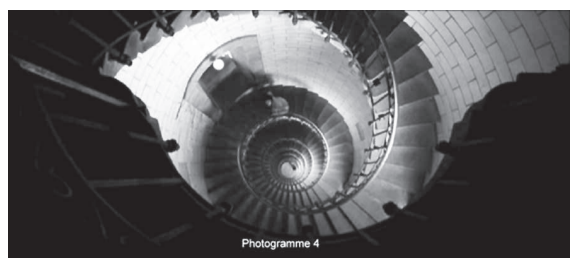

Mathilde(f), filmée de dos, avance et traverse les allées des halles jusqu'à sa rencontre avec Élodie, qui après qu'elle se soit présentée s'éloigne d'elle à toute vitesse. C'est alors que commence la poursuite de Mathilde(f) à travers les étalages, les vendeurs et le public. Un travelling parallèle filme l'angoissante course de la jeune femme pour rattraper Élodie et l'interroger au sujet de son mari. Cette scène, permettant à nouveau de visualiser la persévérance et l'énergie de Mathilde(f), est transformée dans le scénario puisque Japrisot situe la rencontre tranquille entre les deux femmes à l'intérieur de sa voiture où Mathilde(r) a pris rendezvous avec Élodie (LDF, 185-189).
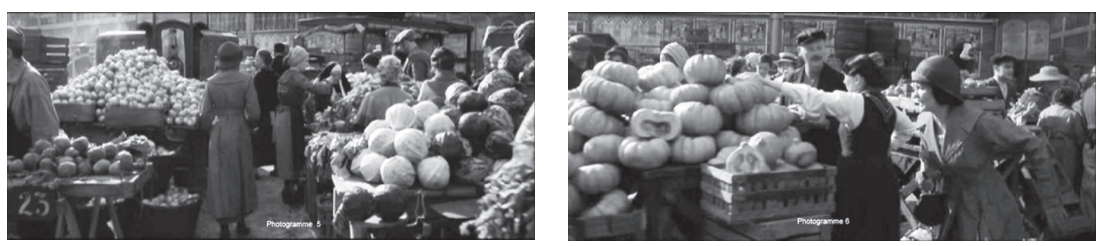

Nous signalerons enfin l'avant-dernière scène du film qui reproduit les instants précédant sa rencontre avec Manech, amnésique dans une maison de santé. Ce «long dimanche de fiançailles » se termine par un plan fixe de la caméra en position de plongée qui filme à contre-jour Mathilde(f) de dos, parcourant tranquillement le jardin situé au-devant du bâtiment, traversant calmement le couloir de la maison plongé dans la pénombre, puis se dirigeant vers la lumière dorée du dehors. Jeunet ne filme pas son visage, il préfère fixer toute l'attention sur sa marche irrégulière, une allure sereine, rassurée et constante, comme elle-même. Tandis que la jeune fille s'avance vers la porte par laquelle entre la lumière dorée du dehors, le contraste entre la luminance forte du fond et la luminance nulle ou faible portée sur Mathilde(f) provoque la lente progression d'une ombre où l'on perçoit la tristesse et la mélancolie qui, pendant de longues années, a été la sienne. 

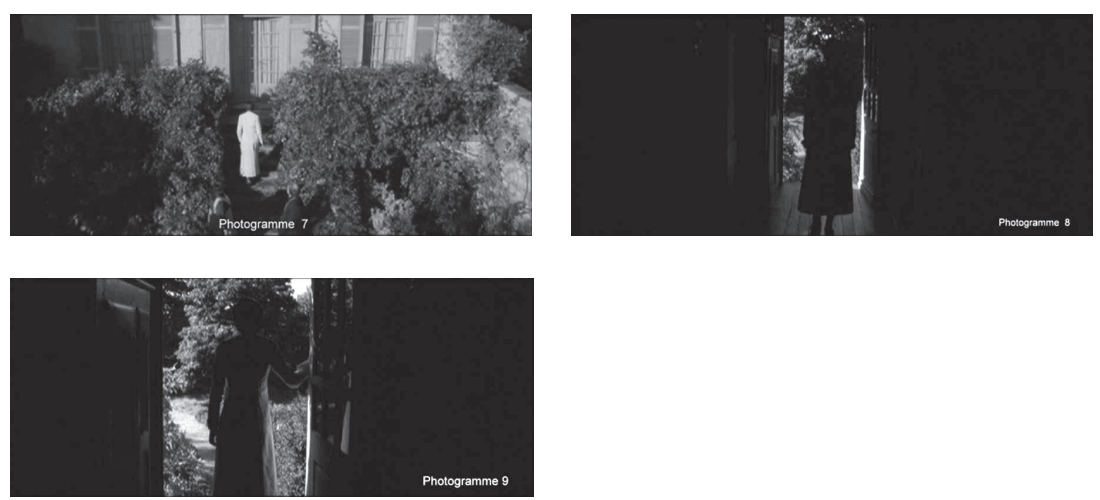

Sur leur hypotexte, Jeunet et Laurant ont ajouté les deux premières scènes puis modifié la troisième et la quatrième. Ce faisant, ces scènes focalisées sur le corps de Mathilde(f) en déplacement renforcent la personnalité de la jeune femme (r), dont les piliers sont l'énergie, la volonté et la confiance. Certes, Japrisot accorde lui aussi ces trois «vertus» à son personnage, mais, cloué qu'il est à sa chaise roulante, ces vertus s'effacent perdant toute existence.

Concernant également son handicap et son estime de soi, Mathilde(r) n'est, selon la narratrice, apparemment victime ni d'elle-même ni d'autrui :

Son fauteuil, par exemple, qu'on ne la plaigne pas d'y être enchaînée, très souvent elle l'oublie. Elle se déplace comme elle est habituée à se déplacer, elle n'y pense guère plus que Véronique a ses jambes. Et si elle y pense, c'est que son fauteuil est lié à tous les souvenirs de Manech $(L D F, 215)$.

Son orgueil efface toute trace de compassion sur soi-même ou de recherche d'indulgence des autres. Il ne l'empêche pas pourtant de se voir parfois différente des autres femmes à cause de la dystrophie musculaire de ses jambes. L'allusion faite à Véronique Passavant ${ }^{31}$ est très significative car le lecteur s'apitoie de cette "féminité handicapée ». Autrement dit, si cette différence n'avait jamais été un fantôme dans son esprit, Mathilde(r) ne l'aurait jamais ressentie comme telle, et la narratrice en aucun cas dévoilée. Retenons

\footnotetext{
31 Véronique Passavant est la maîtresse de Bastoche (ou Eskimo, pseudonymes de Kléber Bouquet), l'un des cinq condamnés à mort pour mutilation volontaire.
} 
que, dans l'imaginaire collectif, la marche d'une femme constitue une image très sensuelle. À ce propos citons, Bertrand Morane, le protagoniste de L'homme qui aimait les femmes (François Truffaut, 1977), lorsqu'il affirme : « Pour moi, rien n'est plus beau à regarder qu'une femme en train de marcher». La comparaison établie par elle-même avec la femme non handicapée révèle donc sa propre perception de posséder une caractéristique physique qui marque sa différence avec les autres femmes, lesquelles traversent les rues sur deux jambes non poliomyélitiques à une allure cadencée.

Le lecteur ne peut qu'être sensible au fait que Mathilde ressent une identité et à une féminité différentes, sauf lorsqu'elle se trouve entre les bras de son fiancé à la citation suivante laquelle, renvoyant à l'intimité de Mathilde et de Manech : «Elle retrouve aussi le goût du vent salé, la vue des dunes, par-delà les fenêtres, où Manech l'embrassait, serrée fort contre lui, désireuse, désirée, pareille aux autres » $(L D F, 150)$. Avec Manech, Mathilde jouit pleinement des sensations et des sentiments, c'est alors qu'elle prend conscience de n'avoir ni un corps ni une féminité « handicapés ». Elle désire autant qu'elle est désirée. Elle se trouve par conséquent sur un plan d'égalité avec les femmes à mobilité non réduite, car les sensations éprouvées avec les étreintes de Manech lui démontrent que l'amour et la passion n'établissent aucune distinction entre femmes handicapées et femmes non handicapées. Néanmoins, si la narratriceMathilde(r) introduit cette réflexion, c'est parce qu'elle n'ignore pas la discrimination, le dédain affiché par Vitré et Fries lorsqu'ils affirment qu' « en situation d'infirmité, sexualité et affectivité, [les femmes] sont, la plupart du temps, niées dans la société [...], insidieusement castratrice en ce qui concerne nos attentes affectives et sexuelles $»^{32}$.

La suppression de ces scènes, si significatives dans le scénario, modifie manifestement la sexualité de Mathilde(r), et du coup sa personnalité épargnée par ces traumas.

D'autre part, l'introduction par les scénaristes de deux scènes où Mathilde utilise son handicap et un fauteuil roulant pour en obtenir les bonnes grâces de Pierre-Marie Rouvière ${ }^{33}$ ajoutent au personnage un penchant pervers et malhonnête. «La fin justifie les moyens ", c'est pourquoi Mathilde(f) qui se rend chez l'avocat pour lui demander de réaliser certaines démarches relatives à la disparition de Manech, obtient son aide après l'avoir ému en faisant état de l'aggravation de sa santé. Après avoir accepté, Pierre-Marie, confus et

\footnotetext{
${ }^{32}$ Françoise VATRE et Vincent FRIES, «Les oubliés de l'amour », in Michel MERCIER, L'identité handicapée, Namur, Presses Universitaires de Namur, 2004. p. 81.

${ }^{33}$ Pierre-Marie Rouvière est l'avocat de la famille dans le roman ; il devient dans le film l'ex avocat de son père et son exécuteur testamentaire.
} 
préoccupé par cet état physique, lui assure : « Ça me fait bien de la peine de voir que ton état s'est dégradé, malgré les massages et je... Bon ! D'accord... je vais voir ce que je peux faire pour toi» $(31: 12$ - 33:16). Mathilde(f) n'essaie pas de le rassurer, puisqu'une fois sa confiance gagnée et ses propos échangés, elle s'empresse de quitter avec empressement le bureau en négligeant la gentillesse et les soucis de son tuteur. Elle se fait même provocatrice lorsque, sortant de l'ascenseur, sous le regard de deux voisins qui aimablement lui ouvrent la porte et font le geste de l'aider, elle se lève sans rien dire, prend son fauteuil insolemment puis descend les deux marches.
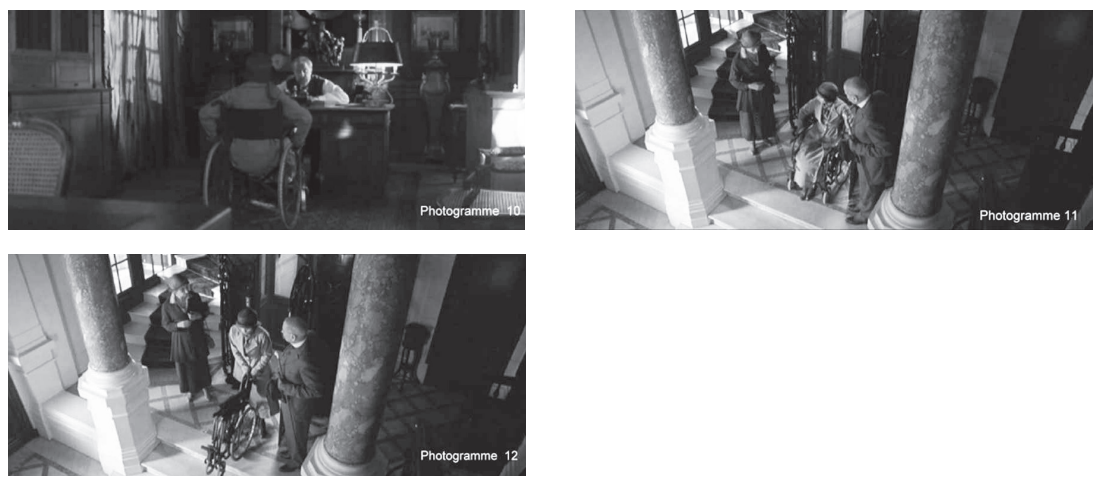

La deuxième scène situe Mathilde(f) aux Archives de l'armée où elle se rend avec Rouvière pour chercher des documents officiels concernant les batailles auxquelles Manech a participé dans le département de la Haute-Saône (38:15 - 41:52). De nouveau la jeune femme utilise le fauteuil roulant pour se déplacer, en prévision de continuer la farce de son infirmité et de pouvoir ainsi atteindre ses objectifs. Une fois de plus, la jeune femme fait montre de méchanceté, d'indifférence face à la terrible angoisse qu'éprouver Rouvière. L'obstination que met Mathilde(f) à éclaircir la mort de Manech ne la fait reculer devant rien, son acharnement sans scrupule s'accomplit aux dépens de quiconque.

Mathilde(f) instrumentalise son handicap pour susciter la pitié des autres et ainsi les séduire afin de mieux obtenir leurs faveurs. Ces deux scènes chargent le caractère de Mathilde(r) des teintes assez peu dignes, qui traduisent sa ruse et sa sournoiserie. 
Signalons enfin une scène où elle va à Daniel Esperanza ${ }^{34}$ pour le rappeler à l'ordre par l'interpellation suivante : « Je suis boiteuse, pas idiote » (17:50). La manière de fulminer contre son interlocuteur en se référant à son handicap permet d'entrevoir le trauma causé par sa jambe semi-rigide.

Concernant l'influence du handicap sur la personnalité de Mathilde, on a pu constater que les modifications de Jeunet et Laurant sur leur hypotexte obéissent à une finalité esthétique, car les différents plans utilisés sont destinés à mettre en relief son allure boiteuse. Les plans fixes larges situent Mathilde et sa jambe poliomyélitique dans l'échelle du contexte spatial, tout en l'éloignant de son destin, de même que les plans rapprochés en mouvement signalent et accentuent chacun de ses pas, marchant ou courant. Esthétiquement, dans le film Mathilde(f) est facilement reconnaissable par sa marche irrégulière dans des lieux tortueux et irréguliers. Son courage et son obstination face aux obstacles physiques seraient ainsi comparables à sa volonté et sa fermeté face aux obstacles de diverse nature qui font obstacle à sa recherche de Manech.

Mathilde(r) et Mathilde(f) possèdent la même vitalité et une énergie physique et morale identique. Il existe pourtant certains traits propres à la personnalité de Mathilde(r) comme son orgueil tiré de son corps handicapé surmontant les barrières et comme sa sexualité «non handicapée » qui occupent une place importante dans le film. Les scénaristes s'appuient sur son corps et son handicap pour souligner certains traits de la personnalité de Mathilde(f) tels que la confiance, la volonté, la ténacité, la résistance et la souffrance physique et morale. D'autre part, et contrairement au roman, en raison de l'instrumentalisation de son handicap, on observe chez elle un endurcissement de son caractère lorsque, conduite par un égoïsme excessif, elle a recours à des ruses moralement discutables.

\section{Caractéristiques de la personnalité de Mathilde à partir sa dimension intime et sociale}

Mathilde, l'adolescente introvertie passionnée pour les chats et amoureuse de Manech, devient une jeune fille dont la dimension intime et sociale ne subit aucune variation jusqu'à la disparition de son fiancé. Cet événement bouleverse sa réalité quotidienne et sa stabilité émotionnelle, modifiant

\footnotetext{
${ }^{34}$ Daniel Esperanza, ancien sergent qui reçoit l'ordre de conduire les cinq condamnés à mort pour mutilation volontaire jusqu'à la tranchée «Bingo Crépuscule ». Après la guerre, il donne rendezvous à Mathilde pour lui raconter les faits de cette nuit du 6 janvier 1917 : les cinq soldats seront ensuite envoyés vers le no man's land.
} 
profondément certains traits de sa personnalité. Selon Pervin et John, la personnalité « représente les caractéristiques de la personne auxquelles renvoie sa manière habituelle de sentir, de penser et de se comporter $»^{35}$. Effectivement, la personnalité de Mathilde subit un bouleversement impressionnant à la suite de la mobilisation de son fiancé : elle ne sent, ni pense, ni se comporte plus jamais comme naguère.

Examinons la personnalité de Mathilde sur plusieurs plans : sa quête, sa liaison affective et intime avec Manech, ses rapports familiaux, sa relation avec les animaux, ses goûts artistiques.

\subsection{Mathilde, l'enquêteuse}

C'est fondamentalement vis-à-vis de sa quête que l'on constate le mieux la combinaison d'émotions, d'attitudes et de comportements formant la personnalité de Mathilde. Celle-ci s'est certainement forgée à cause de son handicap et de sa lutte permanente pour surmonter toutes sortes d'obstacles physiques et sociaux. De ce fait, Mathilde(r) et Mathilde(f) ont en commun un optimisme et une détermination mis en évidence, dès le début, par les narratrices des récits narratif et filmique :

Et puis, Mathilde est d'heureuse nature. Elle se dit que si ce fil ne la ramène pas à son amant, tant pis, ce n'est pas grave, elle pourra toujours se pendre avec $(L D F, 31)$.

\section{VOIX OFF}

Et puis Mathilde est d'heureuse nature. Elle se dit que si le fil ne la ramène pas à son amant, tant pis, c'est pas grave, elle pourra toujours se pendre avec $(12: 31-12: 40)$.

Dans la quête de la jeune femme, Jeunet et Laurant conservent également de leur hypotexte le versant menteur de Mathilde(r), mais dans des scènes différentes. À ce propos, le commentaire de la narratrice, lorsque Pierre-Marie fait promettre à Mathilde de garder le secret des informations obtenues grâce à un officier d'état-major, est très révélateur : "Menteuse comme elle se connaît, Mathilde promet sans hésitation » $(L D F, 130-$ 131). Bien que la jeune femme acquiesce tout de suite et catégoriquement, l'intervention de la narratrice nous permet de conclure que ce mensonge n'est

\footnotetext{
${ }^{35}$ Lawrence A. PERVIN et Oliver P. JOHN, La personnalité : de la théorie à la recherche, Québec, Éditions du Renouveau Pédagogique INC, 2005, p. 4.
} 
ni involontaire ni occasionnel. Dans le film, les mensonges de Mathilde(f) se réduisent à l'utilisation du fauteuil roulant; cependant on mettra en exergue les conséquences des mensonges de la jeune femme lorsqu'elle feint une aggravation de sa maladie.

Cependant, il y a certains traits de personnalité qui diffèrent entre les deux textes. Mathilde( $\mathrm{r}$ ) semble en général beaucoup plus calme que Mathilde(f). La scène de l'hôpital où Mathilde vient interroger Espéranza présente une Mathilde(f) beaucoup plus contenue :

Elle se penche en avant et le presse d'une voix douce : « Je vous en prie, où l'avezvous vu ? Racontez-moi. Que lui est-il arrivé ?» $(L D F, 37)$.
MATHILDE

Dites-moi ce qui arrivé à Manech, je vous en prie 3:22)

La suite de questions adressées à Espéranza lors de leur rencontre peigne une Mathilde(r) inquiète des nombreuses digressions du sergent malade, mais fondamentalement à cause de son impatience de connaître la vérité. Les scénaristes transforment les questions en une seule exclamation traduisant sa rigueur et son exigence de précision pendant l'entretien.

De retour en train à Cap Breton, la narratrice accompagne le silence de Mathilde(r) d'une nouvelle confidence très éclairante sur sa personnalité : « elle est submergée d'orgueil et de reconnaissance pour elle-même » $(L D F$, 149). Cette intervention de la narratrice, supprimée dans le film, est très éloquente, car elle met en relief la fierté et la confiance en elle-même de Mathilde dans une entreprise à laquelle, incrédules, ses proches s'opposent depuis le début.

Cependant, sa recherche traverse parfois des phases très ingrates et décourageantes par son absence de résultats, c'est alors qu'intervient le tempérament colérique de Mathilde(r) : " jusqu'à ce qu'un soir, excédée, elle fasse valser les assiettes et les verres d'un coup de poing sur la table : «l'Opération Peau de Chagrin » $(L D F, 214)$. Cette Mathilde désespérée et violente est absente du film, puisque les scénaristes préfèrent mettre en scène une Mathilde plutôt modérée et contenue dans ses instants de faiblesse, de rage ou d'impuissance.

Dans ce roman où l'histoire, le suspense et les sentiments s'enchaînent, l'enquête policière menée par Mathilde qui dure quatre ans fait ressortir son optimisme et sa détermination, deux qualités communes aux deux Mathilde. Cependant, on peut observer des différences comme l'irritabilité, le 
désespoir et l'impétuosité de Mathilde(r) face à la modération et l'assurance de Mathilde(f).

\subsection{Mathilde, la fiancée}

Si la quête de Mathilde constitue le thème principal de l'argument des deux récits, cette quête est inspirée par son amour pour Manech. De ce fait, la construction du roman et du film recourant à des flashbacks permet aux lecteurs et aux spectateurs de découvrir l'histoire des deux jeunes gens, puis de comprendre l'importance de l'amour de Mathilde pour Manech.

Jeunet et Laurant ont transformé l'histoire d'amour imaginée par Japrisot. Cette adaptation détermine essentiellement deux Mathildes singulières, bien qu'initialement les deux récits accordent la même importance au romantisme, au sentimentalisme, à la passion, puis à la volupté sexuelle.

En matière de fidélité et de loyauté relevons des différences assez évidentes entre les deux «veuves blanches ». Dans le roman après la disparition de son fiancé, Mathilde(r) se questionne sur le respect à son engagement pris envers Manech :

Elle se demande si, ayant deux enfants de lui, elle pourrait vouloir oublier Manech. Elle ne sait pas. Elle se dit que non, mais aussi, bien sûr, que Thérèse Gaignard $^{36} \mathrm{n}$ 'a pas, elle, un père qui gagnait déjà beaucoup d'argent avant la guerre et en gagne encore plus maintenant, dans les villes anéanties $(L D F, 105)$.

Face à ce dilemme, Mathilde(r) demeure très lucide, réaliste et pragmatique puisqu'elle comprend parfaitement que, face à la situation précaire des autres veuves de guerre, son excellente position économique favorise son indépendance et sa fidélité à la mémoire de Manech. Sur ce point, citons François Cochet pour qui «Le rôle des femmes dans la Grande Guerre se mesure aussi à l'aune de la souffrance [...]. La guerre et ses difficultés matérielles posent surtout la grave question de l'absence et de la mort $»^{37}$. Grâce aux historiens, nous savons qu'un pourcentage élevé de veuves sans emploi avec un ou des enfants à charge chercha à se remarier, seule issue possible de leur misère. Japrisot nous révèle cette donnée historique très importante, mais ce n'est justement pas le cas de Mathilde(r), qui peut compter sur la protection économique de son père.

\footnotetext{
${ }^{36}$ Thérèse Gaignard est la femme de Francis Saignard, l'un des cinq condamnés avec Manech.

${ }^{37}$ François COCHET, 1ère Guerre mondiale : date, thèmes, noms, Paris, Studyrama, 2001, p. 71.
} 
La suppression de la réflexion précédente de la narratrice dans le film nous permet de considérer la fidélité de Mathilde(f) comme inébranlable puisque jamais l'idée d'avoir une autre relation sentimentale ne lui vient à l'esprit.

Cependant, les deux Mathilde(s) se rapprochent en matière de loyauté. Le premier des hommes à faire basculer leur loyauté sera M. Cornu, leur masseur $(25: 21-25: 43)$ :

Elle ferme les yeux. Elle se laisse pétrir. Elle imagine que Georges Cornu admire ses formes et n'en peut plus de désir. Une fois, il lui a dit : «Vous êtes drôlement bien bousculée, mademoiselle. Et je peux vous assurer que j'en vois.» Ensuite Mathilde ne savait plus si elle devait l'appeler : Mon cher Georges, Mon très cher Georges ou Jojo $(L D F, 86)$.

\section{VOIX OFF}

Mathilde a besoin de soins quotidiens pour ses jambes. Depuis l'armistice, George Cornu, vice champion de Bretagne de nage libre, vient la masser. Dans les premiers temps, elle était un peu honteuse qu'il la tripote sur tout le corps, même les fesses. Et puis elle s'y est habituée.

Elle ferme les yeux et se laisse faire, tandis qu'un grand moustachu la pétrit vigoureusement.

VOIX OFF

Lorsqu'elle est humeur, Mathilde imagine que Georges Cornu admire ses formes et n'en peut plus de désir. D'ailleurs, un jour il s'est permis de dire :

CORNU

Z'êtes drôlement bien balancée, mademoiselle, et je peux vous assurer que j'en ai tâté quelques-unes.

VOIX OFF

Ensuite Mathilde ne savait plus trop si elle devait l'appeler :

MATHILDE (répétant devant son miroir) Mon cher Georges... mon très cher Georges... Jojo...

Les compliments de Cornu, «illustrés» par ses mains massant ses fesses et ses hanches, excitent des pensées libidineuses Mathilde d'où l'échec de sa loyauté. Dans le film (25:20 - 25:43), cet épisode est beaucoup plus évident grâce aux différentes possibilités qu'offre l'échelle de plans de la caméra : une plongée reprenant les mains de Cornu massant ses fesses, un travelling en avant filmant le visage de Mathilde(f) avec un léger sourire, puis immédiatement après la flatterie du masseur un changement brusque de cadrage 
à travers un gros plan enregistrant les yeux grands ouverts de Mathilde, suivi d'un léger déplacement de ses pupilles vers la droite. Selon la psychologie, l'écarquillement des yeux dénoterait sa surprise ainsi que son mouvement vers la droite témoignerait que son imagination s'est réveillée ${ }^{38}$.
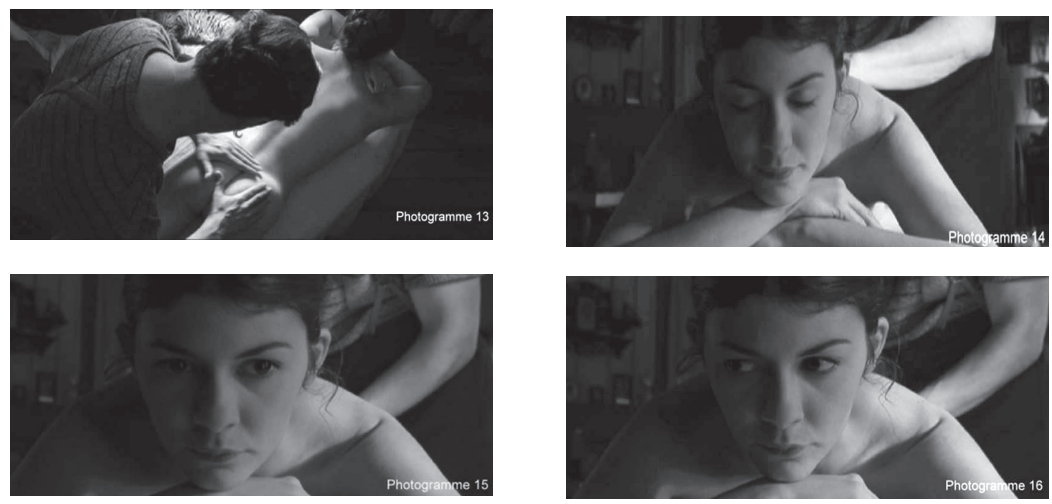

Effectivement, les deux Mathildes vont mépriser la mémoire de leur fiancé lorsque dans la scène suivante au massage, face au miroir et à voix haute, l'une et l'autre essaieront plusieurs expressions, qui oscillent de la formule de politesse au surnom, pour s'adresser au masseur.

Cornu n'est pas le seul homme avec qui Japrisot « tente » Mathilde(r). Le chirurgien new-yorkais Amo Feldmann, est aussi un «fruit défendu» qu'elle parvient à écarter d'elle - au moins dans la réalité, puisque ses rêves, tel qu'on peut le constater plus tard, sont incontrôlables - :

C'est un fiasco sans intérêt, sauf que cessent les douleurs qui la tuaient au niveau des hanches et qu'elle est au bord de tomber amoureuse du chirurgien, mais il est marié, père de deux gamines aux joues rondes, criblées de taches de rousseur, il n'est même pas beau, et comme chacun sait, si l'on oublie des inconnus sans visage qui la tourmentent quelquefois dans des rêveries regrettables, Mathilde ne trompe jamais son fiancé $(L D F, 245)$.

\footnotetext{
${ }^{38}$ David COHEN, Comment décoder les gestes de vos interlocuteurs, Paris, LEDUC, coll. «DEVELOPPEMENT », 2010, p. 123.
} 
Célestin Poux ${ }^{39}$ est «la dernière tentation » de Mathilde. Son apparition dans la ferme annonce un tournant favorable dans la quête et bouleverse la quiétude sensuelle de la jeune fille, comme nous pouvons l'inférer des commentaires de la narratrice :

Quand il entre dans la chambre, il a les joues les plus roses, les yeux bleus les plus candides qu'on ait jamais vus $(L D F, 316)$.

Bien des années plus tard, quand il arrivera a Mathilde de penser à Célestin Poux -ce qui sera somme toute aussi fréquent que de penser à sa propre jeunesse-, ce qu'elle reverra de lui en premier sera ses cheveux blonds et les deux gros ronds de peau rose, bien propres, qu'il gardait autour de ses yeux bleus en débarquant, ce dimanche d'août $(L D F, 267)$.

L'insistance de Mathilde(r)-narratrice montre son attraction pour certains traits du visage de Célestin, qu'elle dépeint avec tendresse à juger par les adjectifs utilisés dans les descriptions.

Grâce aux flashbacks, lecteurs et spectateurs connaissent Mathilde dans les temps heureux de ses fiançailles avec Manech avant que la guerre ne vienne détruire son bonheur. Mais ces temps heureux possèdent également certains épisodes sombres qui font ressortir des sentiments tels que la jalousie. Teverino affirmait que « Là où il n'y a point d'amour, il n'y a point de jalousie $»^{40}$, et, effectivement Mathilde(r), ait l'expérience de cette double postulation douloureuse. La jalousie est, de nouveau, un trait de la personnalité de Mathilde(r) supprimée par les scénaristes, un sentiment qui cependant a pour source son innocence.

Lorsqu'en été 1915 Patty, une Anglaise blonde divorcée, arrive à CapBreton, Manech, irrésistiblement attiré par les invites de la touriste, s'éloigne de sa fiancée. Mathilde est bouleversée par la perte et le partage de l'être aimé, d'où, conséquence de cette infidélité, la rancune, le désir de vengeance et las pensées morbides de Mathilde(r), sentiments qui ne l'avaient jamais auparavant effleurés. De nouveau, c'est l'indiscrétion de la narratrice qui dévoile la face cachée de Mathilde(r) :

Reste à Mathilde, dans son lit, de se délecter des supplices qu'elle voudrait infliger ou voir infliger à la vilaine femme. Un jour, la croisant sur le pont de

\footnotetext{
${ }^{39}$ Ex-soldat fringant et habile, témoin de faits qui entourent la disparition des cinq soldats.

${ }^{40}$ George SAND, Teverino, Babel n437, Arles, Actes Sud, 2003, p. 242.
} 
bois d'Hossegor, elle fonce sur elle avec sa trottinette, lui écrase les pieds et l'envoie se noyer dans le canal ( $L D F, 230-231)$.

Trompée et délaissée, la jeune fille se place au centre d'un triangle formé par la haine, la rancune et la vengeance contre la femme responsable de l'abandon de Manech. Le film néglige ce côté ombrageux et haineux de Mathilde(r). Toutefois, l'apparition de Patty transforme la relation entre les jeunes gens qui jusqu'à cet été s'était limitée à une relation platonique :

C'est là, au cours d'une halte forcée, durant l'été 14, à quelques jours de la guerre, que Mathilde, voulant l'embrasser sur la joue, pour le réconforter, laisse glisser ses lèvres et, le diable aidant, l'embrasse sur la bouche. Elle y prend goût si vite qu'elle se demande comment elle a pu attendre si longtemps et lui, ma foi, il est rouge jusqu'aux oreilles, mais elle sent bien qu'il ne déteste pas la nouveauté ( $L D F, 228-229)$.

Bien que timide, Mathilde(r) possède une évidente initiative dans leur relation sentimentale. Son geste est initialement un signe de protection et de tendresse, mais il devient fortuitement la première expression d'un amour s'acheminant vers le charnel. Jeunet et Laurant transporte l'histoire du premier baiser là une époque où Mathilde et Manech ne sont que des enfants : il ne s'agit que d'un jeu innocent. En haut d'un phare, Mathilde(f) à l'intérieur de la lanterne et Manech dehors s'embrassent plusieurs fois pour de faux à travers les vitres (1:05:49 - 1:05:54). Malgré la naïveté de ces baisers, leur intensité montre l'existence d'un sentiment très vif.

Dans le roman, en matière sentimentale, Mathilde(r) retrouve pleinement Manech après le départ de Patty : «Manech oublie ses scrupules, il recommence à l'embrasser comme elle aime, et un soir aussi, il embrasse ses seins, qu'il trouve très beaux. Entre honte et délice, Mathilde croit mourir » $(L D F, 231)$. Cette scène, notablement modifiée dans le film, complète le portrait de Mathilde(r) qui se donne sans réserve à l'amour physique. Initiée avec bonheur par Manech, déjà expérimenté dans la matière grâce à Patty, la débutante éprouve de nouvelles sensations intimes, lui révélant une sensualité et une sexualité inconnues jusqu'à cet instant. Dans ces instants d'intimité Mathilde(r), profondément troublée par les nouvelles sensations physiques, se débat entre pudeur et excitation.

Toutefois, ce n'est qu' avec la mobilisation de Manech que définitivement sonne la fin de leur relation platonique : 
Manech emporte Mathilde dans la cabane de Croquemitaine, qu'on ne voit plus depuis deux ans, qui doit être sur le front, lui aussi. Il l'étend sur des filets de pêcheurs qu'on appelle des sennes, il la déshabille un peu, elle s'affole un peu, sans oser rien dire tant le moment lui paraît solennel, il l'embrasse partout, elle a le feu aux joues et lui aussi, ensuite elle a mal comme elle l'appréhendait dans ses divagations nocturnes, mais pas si mal quand même, et ensuite encore, elle est bien comme elle l'espérait aussi, et même mieux $(L D F, 232)$.

Consciente de ce qui va se passer, Mathilde(r) se montre troublée dans les préliminaires de leur première relation sexuelle. Cependant, son bouleversement ne l'empêche pas d'être réaliste quant aux actions qui suivront, et de ne pas dramatiser la perte de sa virginité, appréciant le torrent de sensations physiques nouvelles qui parcourent son corps.

Jeunet et Laurant modifient entièrement la narration de la première relation sexuelle des jeunes amants. Cette relation, au centre de la narration, est narrée en deux temps au moyen un renversement chronologique des séquences initiales et finales. Située presqu'au début du film ${ }^{41}(11: 33$ - 12:00), elle constitue la présentation des deux personnages principaux à l'âge adulte. Il s'agit d'une prolepse ${ }^{42}$, manœuvre narrative permettant aux scénaristes d'avancer l'histoire d'amour des protagonistes. Cette scène est esthétiquement très soignée par Jeunet, essentiellement par la grâce de l'éclairage, pour lequel Jeunet utilise des filtres aux tons jaunâtres, presque dorés, pour adoucir les tons du visage de Mathilde(f) et lui accorder un ton clair et éclatant qui traduit le bonheur de ces instants. Un travelling avant et un plan « taille » focalisent sur les amants endormis au lever du jour, l'un contre l'autre, Manech serré contre le dos de Mathilde(f) sa main posée sur son sein. Lorsque Manech retire sa main pour écarter une petite araignée sur sa joue, Mathilde(f) dans son sommeil la repose à nouveau délicatement sur son sein. Les premiers rayons de soleil du jour illuminent le visage heureux et serein de la jeune amante, illuminé d'un léger sourire sur ses lèvres pulpeuses.

Le début de cette scène est introduite in media res du film (1:05:55 1:07:03), à la suite des baisers en haut du phare, ce moyennant un déplacement chronologique. Effectivement, à ces étreintes enfantines succèdent d'autres étreintes beaucoup moins innocentes mais aussi tendres et amoureuses. Jeunet et Laurant mettent en scène le couple d'adolescents jouant au mikado

\footnotetext{
${ }^{41}$ Par contre, dans le roman elle fait partie du huitième chapitre (le roman est divisé en quatorze chapitres).

${ }^{42}$ Par contre, il s'agit d'une analepse par rapport à la scène antérieure, situant Manech au front.
} 
à l'intérieur d'une petite cabane éclairée par une lampe à pétrole. L'éclairage ténu et jaunâtre qui baigne les amants est soigneusement mis au point pour préserver l'intimité de leur première relation intime. Dans le roman, Mathilde(f) embrasse délibérément et tout doucement les lèvres de Manech, qui silencieux éteint ensuite la bougie. Dans cette ambiance apaisante et romanesque, Mathilde(f) se livre à une mystérieuse et suggestive séquence érotique en se déshabillant peu à peu, profitant l'obscurité tandis que Manech enflamme allumette sur allumette qu'elle-même éteint aussitôt. Le contre-éclairage de Manech le transforme presque en une sombre du corps de Mathilde illuminé par la flamme des allumettes. Face à l'image sombre de son fiancé, Mathilde(f) se montre peu à peu sensuellement, résolue et sereine, discrète et pudique, séductrice et réservée. Toutes ces qualités sont mises au service d'un striptease très délicat destiné à provoquer l'acte sexuel. Nous partageons le jugement de Serge Chaumier à propos des intentions de ce lent « dévoilement » ritualisé qui se déroule dans la solitude de la cabane en compagnie de Manech : «ce qui est excitant, c'est l'attente maintenue en état de tension $»^{43}$. Mathilde joue avec l'attente de Manech, et bien qu'inexpérimentée, elle sait comment provoquer par le dévoilement de son corps, rythmé par les clairs-obscurs, une intensification du désir.
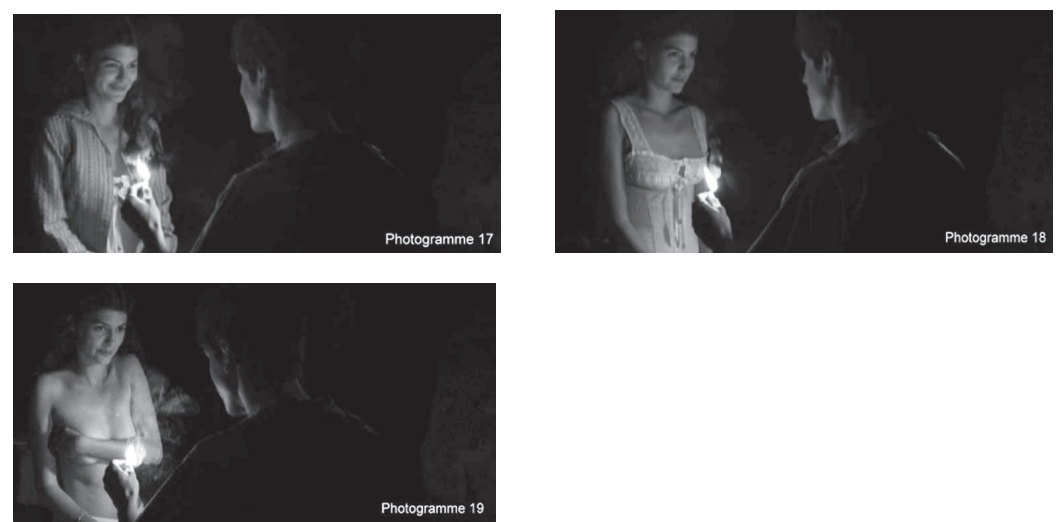

${ }^{43}$ Serge CHAUMIER, L'amour fissionnel : Le nouvel art d'aimer, Paris, Fayard, 2004, p. 300. 
Le lyrisme de ces scènes intimes contraste avec la sobriété passionnelle et sensuelle de Japrisot. Le romancier efface toute trace d'érotisme dans ces scènes d'intimité.

Nous avons précédemment indiqué que la fidélité constituait le trait principal de Mathilde (r), puis que son imagination au cours de ses contacts avec le masseur et le chirurgien avait entamé sa loyauté. Revenons sur ce point : dans les textes narratif et filmique, les rêves de Mathilde l'entraînent au-delà d'une simple relation platonique, la transportant dans un univers d'affrontements, de résistances, d'intimidations et d'abus. La narratrice détaille pour le lecteur quels fantasmes l'habitent avant qu'elle ne s'endorme :

Mathilde, comme son arrière-grand-mère inventée, est une belle coquine. Avant de s'endormir, elle s'imagine dans des situations troublantes, toutes plus invraisemblables les unes que les autres, encore qu'elles tournent toujours autour du même thème simplet : elle est la victime d'un inconnu -elle ne voit jamais vraiment son visage- qui la surprend quelque part en chemise, ne peut résister à la formidable envie qu'il a d'elle, la cajole, la menace, la dénude, jusqu'à ce qu'elle se résigne à l'inévitable ou l'appelle de tous ses vœux. La chair est si forte. Mathilde a rarement besoin d'aller aux ultimes péripéties de ses divagations pour que le plaisir l'emporte, si impératif, si aigu parfois qu'il lui semble s'irradier jusque dans ses jambes. Elle s'enorgueillit de ce plaisir et d'en être capable, qui la rend, pendant quelques poussières d'éternité, pareille aux autres $(L D F, 86-87)$.

Utilisant la technique de marquage sur l'écran de " l'œil fantasmé », Jeunet et Laurant transposent les fantaisies nocturnes de Mathilde(r) dans un film muet de l'époque (25:52 - 26:18). Le changement des plans de Mathilde(f) couchée dans son lit puis du tableau muet truqué révèle par rapprochement le contenu des fantaisies de la «jeune veuve». Sur l'écran Mathilde(f), transformée en héroïne de cinéma, est victime de la séduction forcée d'un agresseur masqué, que l'on peut identifier à Cornu. La séquence du viol s'achève par une fermeture de l'iris de ses yeux sur le visage resplendissant de la jeune fille se masturbant.

Sachant qu'il ne s'agit pas d'un rêve mais d'un fantasme, si l'on s'en rapporte aux interprétations freudiennes des rêves, celui-ci est une « voie royale qui mène à la connaissance de l'inconscient $»^{44}$. De lors on pourrait affirmer que l'inconscient de Mathilde se rebellerait contre sa fidélité, contre sa loyauté, mais surtout contre sa chasteté. Comme les interventions des narratrices faisant

\footnotetext{
${ }^{44}$ Sigmund FREUD, Cinq leçons sur la psychanalyse, Paris, Payot, 1966, p. 38.
} 
part de ces désirs intimes se situent quelques paragraphes après la scène du masseur, il serait peut-être légitime d'interpréter le désir de Mathilde comme une inclination ardente à établir des relations sexuelles avec Cornu, homme très viril débordant sensualité et sexualité qui l'excite. En outre, si l'on se réfère à la théorie jungienne selon laquelle « la fonction générale des rêves est d'essayer de rétablir notre équilibre psychologique à l'aide d'un matériel onirique qui, d'une façon subtile, reconstitue l'équilibre total de notre psychisme tout entier $»^{45}$, on pourrait en déduire que ce fantasme réitératif, au cours duquel Mathilde révèle les penchants de sa libido, lui permettrait de contrebalancer ses troubles émotionnelles dus à l' absence affective et « effective » de son fiancé et amant. Certes, Mathilde reste fidèle à Manech, mais, à considérer ses aventures amoureuses, imaginaires, mais conscientes, elle serait déloyale.

Cependant, la fin de la citation indique que la force de ses fantasmes sexuels introduisant des agresseurs inconnus réveille sa libido. De pair avec son auto érotisme, la voix omnisciente et indiscrète de la narratrice confie la fierté de Mathilde au sujet de sa sexualité, puisque son excitation et la jouissance de son orgasme lui confirment qu'elle n'est ni inférieure ni différente des femmes non handicapées.

Les narratrices des textes filmique et narratif ne font pas la moindre réserve à propos de l'intimité de Mathilde, cependant il faut noter la seule différence notable : dans le roman la narratrice revient plusieurs fois sur le sujet dans deux pages qui se suivent; ce qui pourrait découler du fait que le degré de handicap de Mathilde(r) y est supérieur, d'où ses soucis :

Jamais, depuis l'annonce de sa disparition, Mathilde n'a pu supporter la pensée de son fiancé quand elle se contente. Et il est de longues périodes où elle a honte d'elle-même et se déteste et se jure bien de fermer sa porte aux inconnus. Autrefois, même avant qu'ils aient fait l'amour et durant les mois où il était au front, elle ne se voyait pourtant qu' avec Manech en se donnant du plaisir. C'est comme ça $(L D F, 87)$.

Elle a de très beaux seins. Elle est fière de ses seins, qui sont ronds, lourds, plus doux que la soie. Quand elle en caresse les bouts, elle a bientôt envie d'être aimée. Elle s'aime toute seule $(L D F, 86)$.

Sa fidélité et sa déloyauté mises à part, ces scènes intimes découvrent une jeune femme sans tabous, ayant désacralisé son corps en matière sexuelle

${ }^{45}$ C.G. JUNG, L'Homme et ses symboles, Paris, Robert Laffont, 1964, p. 49. 
; une jeune femme sans préjugés religieux ou moraux, souffrant les carences affectives et charnelles des à l'absence de son fiancé, mais qui sait faire la différence entre la fidélité à son amour et ses pulsions intimes.

Jeunet et Japrisot n'introduisent pas cette séquence dans la scène du film où elle interroge le sergent. En conséquence, ce sentiment de gêne éprouvé par la jeune femme est supprimé, rétablissant ainsi le respect des bonnes manières.

En matière amoureuse, nous avons pu constater que Mathilde(r) et Mathilde(f) sont tout aussi sentimentales dans le domaine intime que passionnelles et voluptueuses. Néanmoins, l'adaptation de Jeunet et Laurant introduisent certaines différences comme la suppression de la jalousie de Mathilde (r) et de son penchant rancunier, vindicatif et pervers. En revanche, Jeunet et Laurant nuancent la part intime de Mathilde(r) en lui conférant une tonalité séductrice, sensuelle et pudique, absente du roman. Roman et film, mettent en scène également, mais avec une tonalité différente la fidélité et la trahison des deux veuves blanches.

\subsection{Mathilde, la fille $v s$ la nièce}

Une des transformations les plus importantes réalisée par Jeunet et Laurant au niveau de l'hypotexte porte sur la situation familiale de Mathilde. Mathilde(f) reste orpheline - et aux soins de ses oncle et tante Sylvain et Bénédicte - après le décès de ses parents dans un accident de tramway à Paris. Par contre, le noyau familial de Mathilde(r) est formé par ses parents, puis par Sylvain et Bénédicte - un couple au service de la famille depuis son enfance - ; au-delà de la maison familiale, son frère, sa belle-sœur et ses neveux complètent le cercle familial plus proche. Dans l'adaptation filmique Mathilde(r) conserve tous ses liens familiaux, tandis que Mathilde(f) est une jeune déshéritée, ce qui double aux yeux du spectateur son courage et ses réussites.

Bien que le plus souvent Mathilde(r) exprime peu ses émotions, la narratrice s'y confond pour préciser ses sentiments envers toute sa famille, en quatre phrases qui résument et spécifient son attachement familial d'une manière exacte et honnête :

Elle est assise à un bout de la grande table, en face de son père, qu'elle aime de tout son cœur. À sa gauche, Maman, qu'elle aime beaucoup. À sa droite, son frère Paul dont elle ne pense pas grand-chose, sinon qu'il est supportable, et sa ni belle ni sœur, Clémence, qu'elle ne supporte pas. Les deux affreux, Ludovic 
et Bastien, huit et six ans de turpitudes, sont depuis longtemps en train de faire pipi au lit $(L D F, 141)$.

Grâce à la voix de la narratrice, on accède à la palette des sentiments éprouvés par Mathilde(r) envers sa famille. Elle n'a pas un sens de la famille très élevé : seuls Son père et sa mère sont objets d'affection, son frère ne provoque en elle que de l'indifférence, l'aversion à sa belle-sœur de l'aversion, quant à ses neveux ils l'exaspèrent. En tout cas, son « asepsie émotionnelle » pour ses proches parcourt quasiment tout le roman et touche presque tous les personnages. Seul, quelques détails nous sont révélateurs de son affectivité envers les siens :

Un bisou sur la joue, qui sent la lavande et le tabac, il se redresse. Quand elle le regarde, il est en train de ramasser son manteau de pluie jeté sur un fauteuil $(L D F, 139)$.

Elle veut qu'il [son père] se rapproche encore, qu'il la prenne dans ses bras. Il la prend dans ses bras. Lui aussi sent l'eau de lavande et le tabac blond, mais elle aime bien, c'est rassurant $(L D F, 145)$.

À la sensibilité sensorielle de Mathilde(r), on pourrait ajouter sa finesse sensorielle lui permettant d'identifier des odeurs communes à son père et à son fiancé, plus exactement l'odeur de son père lui rappelle celle de son amant. Selon Léon Deneb, « el perfume es recuerdo; símbolo de la memoria y presencia; símbolo de la conciencia $»^{46}$. Le parfum est donc déclencheur de souvenirs et d'émotions. C'est donc au lecteur de pointer ces quelques manifestations émotionnelles de Mathilde(r) vis-à-vis son père. En général, Mathilde(r) est extrêmement réservée et peu causante. Les dialogues sont exceptionnels, et lorsqu'ils sont transcrits, ses phrases courtes et souvent tranchantes mettent en relief son caractère abrupt.

Son laconisme verbal est complété par un langage non verbal très significatif : un geste pour protester contre Daniel Espéranza $(L D F, 36)$ ou un petit soupir pour interrompre ses auto-compassions inutiles $(L D F, 36)$, sont parfois suffisants pour-communiquer avec autrui.

Dans le film, Sylvain et Bénédicte, qui occupent la place réservée à ses parents dans le roman, reçoivent peu de témoignages d'affection de la part de leur nièce, bien que ceux-ci lui manifestent constamment leur dévouement. Une des séquences les plus mordantes est celle où Mathilde(f) renvoie aimablement

${ }^{46}$ Léon DENEB, op. cit., p. 194. 
Bénédicte avec un « Merci Bénédicte » (48:23 - 49), après que celle-ci, sous la pluie, lui a apporté des biscuits alors qu'elle est en réunion avec M. Fouine. Ce « merci » est d'autant plus blessant qu'il est suivi du regard fixe et réprobateur qu'elle lance à la vieille dame quand celle-ci, sans le faire exprès, mouille avec son parapluie certains documents déposés sur la table.

Mais c'est surtout, Pierre-Marie Rouvière qui, malgré son caractère affable et affectueux, est traité avec froideur et dédain dans toutes les scènes du film où il apparaît avec elle. Est particulièrement frappante l'attitude grossière montrée envers celui-ci lorsque Mathilde(f) détourne brusquement son visage quand il se rapproche pour l'embrasser, après qu' elle l'a embrassé une première fois (31:02). Dans le roman cette scène est absente, son contenu trouve son équivalence dans une conversation téléphonique, dont on peut extraire les répliques suivantes : « «Il faut que je t'aime beaucoup, Matti. Beaucoup «. Et il raccroche » $(L D F, 318)$ et « « Je te répète qu'il faut que je t'aime beaucoup, Matti, beaucoup «. Elle lui dit qu'elle l'aime aussi » $(L D F, 320)$. Mathilde(r) répond avec gentillesse aux mots aimables de l'avocat lorsqu'il lui déclare son amour. Ainsi, l'amabilité et la douceur de Mathilde(r) contrastent avec l'impolitesse et le despotisme de Mathilde(f).

En modifiant le noyau familial de Mathilde(r), les scénaristes modifient également sa personnalité. Particulièrement remarquable est la conduite hautaine et arrogante de Mathilde(f) avec ses oncles et son tuteur. Pourtant, aucun des trois personnages ne subit les mépris de Mathilde(r) dans le roman, et, bien que froide et distante, elle n'est jamais ni ingrate ni impolie. Par contre, Mathilde(f) fait constamment preuve d'une absence totale de sensibilité affective vis-à-vis de ceux qui s'occupent d'elle et dont preuve envers elle d'affection.

\subsection{Mathilde, l'amoureuse des animaux}

La sobriété dans la communication verbale de Mathilde dans les relations humaines contraste avec sa communication avec ses chats et avec Pois Chiche, le chien de Bénédicte, notamment dans la solitude de sa chambre, espace privé transformé en refuge émotionnel. Mathilde chaque nuit aime à s'entourer de ses animaux pour récapituler les progrès accomplis et leur faire part verbalement de ses émotions et de ses réflexions.

La froideur émotive de Mathilde(r) avec les hommes laisse la place à son affection avec ses mascottes, qui fréquemment sont comblés de caresses et de compliments et, si nécessaire, de réprimandes, comme l'attestent les citations suivantes : 
Ses chats et ses chattes regardent Mathilde jeter les morceaux de papier dans le feu de la cheminée. Elle leur dit : «Tenir sa langue. Voilà un conseil intelligent, pour une fois. Ne croyez-vous pas que j'ai raison de me méfier précisément de celles ou ceux qui me le donnent? » $(L D F, 156)$.

Mathilde jette la lettre de Véronique Passavant dans coffret, referme le couvercle avec précaution, de peur de réveiller une angoisse qui dort, et elle dit à Voleur et Maître Jacques : «Si vous descendez tout de suite de cette table, je consens à vous faire, à vous seuls, une confidence ». Et comme les chats ne bougent pas, elle ajoute, sèchement : «C'est une confidence très confidentielle ». Ils la regardent avec des yeux sans émotion, étrangement fixes, étrangement neutres - on jurerait des yeux de chat — puis, sans se presser, ils vont de concert, sur leurs pattes douces, au même bord de la table et sautent à terre $(L D F, 157)$.

Ses chats et ses chattes deviennent les seuls confidents des progrès de sa recherche, ils sont les témoins de ses sentiments face aux réussites et aux échecs. C'est à eux — ou éventuellement à son père — qu'elle confie ses peines et ses joies.

Sa préférence pour les chats est très significative si on s'en rapporte à une interprétation symbolique du chat puisque ceux-ci évoquent dans l'imaginaire collectif occidental l'indépendance et la protection, la douceur et le sentimentalisme, la clairvoyance et l'ingéniosité, exactement toutes les qualités et capacités que Mathilde possède dans sa quête pour retrouver Manech ${ }^{47}$.

Japrisot et Jeunet-Laurant accordent la même importance à cette communication de Mathilde(f) avec ses animaux, qui contraste avec sa difficulté à interagir socialement avec d'autres personnes.

\subsection{Mathilde, l'artiste}

Lorsque Jeunet et Laurant transforment les goûts picturales de Mathilde(r) en goûts pour la musique, ils semblent avoir suivi les considérations de Mme de Staël lorsque met dans la bouche de Corinne cet aveu : « La peinture ne saurait se contenter d'une expression aussi rêveuse et aussi vague que celle des sons ${ }^{48}$. Mathilde(r) troque sa toile et ses pinceaux contre un tuba dans le

\footnotetext{
${ }^{47}$ Cf. Montserrat ESCARTÍN GUAL, Diccionario de símbolos literarios, Barcelona, PPU, 1996, 149 .

${ }^{48}$ Germain de NECKER, Corinne ou l'Italie, Paris, Gallimard, coll. « Folio Classique », 1992, p. 225.
} 
film, deux expressions artistiques complètement différents mais qui permettant également de manifester ses pensées.

Pour Mathilde(r), la peinture est non seulement une activité artistique délassante pour son esprit épuisé par le «veuvage » et par les multiples démarches enquêteuses, en plus, la toile représente fondamentalement son principal moyen d'expression, ce qui témoignerait à nouveau de sa difficulté à établir des relations humaines et des rapports de communication. Il est difficile de remonter aux origines de son isolement social, cependant Michel Mercier rappelle que « le handicap physique [...] pose des problèmes sérieux dans le champ de la relation interpersonnelle, de la vie affective et de la sexualité $»^{49}$.

Par ailleurs, son admiration pour J.-F. Millet, et son goût qui la porte à peindre exclusivement des fleurs selon la technique réaliste, traduisent son monde intérieur, ainsi que le révèle la narratrice : "Pour elle, les fleurs de Millet resteront tendres et cruelles et vivaces dans la nuit des temps » ( $L D F, 83-84)$. Les adjectifs utilisés pour définir les fleurs peintes par Millet pourraient être également les principaux traits définitoires de la personnalité de Mathilde(r), la délicatesse, la dureté et la vitalité. Symboliquement, les fleurs évoquent l'univers féminin et l'amour, d'autre part, son attrait pour les fleurs pourrait traduire, de ce point de vue, l'espoir de retrouver Manech, car les fleurs représentent dans l'imaginaire collectif l'espérance.

Ses préférences en matière de couleur pour peindre ses fleurs sont également très révélatrices de son caractère si on se rapproche de sa portée symbolique. Le blanc qui « signifie l'absence $»^{50}$ se rapporterait évidemment à la disparation de Manech ; le rouge, «symbole fondamental du principe de vie, avec sa force, sa puissance et son éclat $\aleph^{51}$ renverrait à la vitalité, au courage et à la force physique et émotionnelle de Mathilde(r) mis en œuvre dans sa quête ; le bleu, symbole du « regard [qui] s'y enfonce sans rencontrer d'obstacle et s'y perd à l'infini $»^{52}$, représenterait le désir de vérité de Mathilde(r) ; puis finalement son refus du jaune, couleur « intense, violent[e], aigu[e] jusqu'à la stridence ${ }^{53}$, associée au mensonge et aux traîtres, exprimerait son mépris face à tous ceux qui ont condamné injustement les cinq soldats et les ont livrés au hasard des fusils allemands dans le no man's land.

\footnotetext{
${ }^{49}$ Michel MERCIER, L'identité handicapée, Namur, Presses Universitaires de Namur, 2004, p. 23.

${ }^{50}$ Jean CHEVALIER et Alain GHEERBRANT, Dictionnaire des symboles, Paris, Robert Laffont, 1982, p. 99.

${ }^{51}$ Ibid., p. 659.

${ }^{52}$ Ibid., p. 101.

${ }^{53}$ Ibid., p. 425.
} 
Jeunet et Laurant troquent la toile, les peintures et les pinceaux pour le tuba, un instrument au son grave qui musicalement représente le sérieux et la rigueur, la sérénité et la constance. Le choix des scénaristes n'est, sans aucun doute, pas aveugle puisque ces indications se trouvent également dans la personnalité de Mathilde(f), ainsi que nous avons montré plus haut.

À propos de l'instrument joué par Mathilde(f), on constate de prime abord qu'elle n'interprète jamais une mélodie : son tuba n'émet que des sons graves. Loubet-Poëtte soutient que

dans l'impossibilité de communiquer sa douleur par un discours construit, le personnage utilise ces sonorités qui, à défaut de signifier, jouent sur les sensations et qui s'apparentent à des plaintes ou des soupirs, d'autant plus marquées que le rendu de cet instrument est par nature « anguissant $»^{54}$.

Le caractère indicible de son expérience et de son ressenti trouverait se manifesterait au moyen du tuba. D'autre part, ces notes isolées pourraient être rapprochée de l'appel mystérieux des marins au moyen de conques ; identification est d'autant plus soutenable lorsqu'elle joue face à la mer, au bord des falaises.

Le besoin de communication avec les personnes, le désir d'extérioriser sa souffrance et de s'en défaire, se canalise à travers la peinture et la musique. Ces deux activités montreraient son caractère solitaire et individualiste. D'autre part, il s'agirait également d'une manière de se sentir mieux et d'apaiser l'intensité de ses émotions.

\subsection{Mathilde, « la fiancée veuve »}

Pour compléter la personnalité de Mathilde, nous pouvons nous pencher sur ses relations avec les personnages qui apparaissent tout au long de sa quête et contribuent à compléter son portrait. Mathilde se présente à eux comme la fiancée d'un patriote mort au front, comme une «veuve blanche».

Le premier à faire son apparition est Daniel Espéranza. Sa conversation avec Mathilde permet de saisir immédiatement le caractère réservé et contenu

\footnotetext{
${ }^{54}$ Vanessa LOUBET-POËTTE, L'énonciation cinématographique. Caractéristiques et méthode(s) d'analyse d'une énonciation artistique audio-visuelle dans les longs métrages de Jean-Pierre Jeunet. Delicatessen, La Cité des enfants perdus, Alien Resurrection, Le Fabuleux destin d'Amélie Poulain, Un long dimanche de fiançailles, Tome 1, Thèse pour le Doctorat en Linguistique française. Sous la direction de M. le Professeur Jean-Gérard LAPACHERIE, 2010, p. 373.
} 
de la personnalité de Mathilde(r) ; on pourrait même dire, son caractère réprimé dans la manifestation publique de ses sentiments. En fait, pendant toute la conversation, Mathilde(r) s'efforce de maintenir son calme malgré les mauvaises nouvelles. Dans le roman, la narratrice revient plusieurs fois sur les efforts de la jeune fille pour s'empêcher de pleurer devant le militaire :

Mathilde ne veut pas pleurer $(L D F, 38)$.

Mathilde a un hoquet. Elle regarde cette main, elle la regarde sans pouvoir articuler un mot. Elle ne veut pas pleurer $(L D F, 39)$.

Et Mathilde demande ce que Manech ne voulait pas voir et elle se retient de pleurer $(L D F, 44)$.

Le mal qu'on lui fait la regarde. Elle ne pleure $(L D F, 52)$.

Cette insistance est très révélatrice, car elle montre la force de sa volonté et le contrôle, qu'elle exerce même sur ses émotions afin de ne pas montrer en public ce qu'elle ressent intimement. Cette interdiction vise à interdire toute intrusion dans son intimité, dans ce que Goffman désigne comme «les réserves du moi ${ }^{55}$, à savoir, ces domaines où l'on ne désire pas que l'autre y pénètre.

Elle s'abstient non seulement d'extérioriser sa peine, mais encore sa révolte intérieure : « «Et les fusiller « veut savoir Mathilde, et si son Manech était un des cinq, et elle crie, maintenant, et elle s'entend crier mais elle est sans voix » $(L D F, 38)$. Sa circonspection n'obéit pas au désir de préserver sa dignité ou de montrer sa faiblesse, il s'agirait plutôt d'une attitude consciente propre à refouler son expressivité émotionnelle. Ce cri intérieur, comme ses larmes, l'ont accompagnée dès qu'elle a appris la perte de Manech, dès le début de son enquête.

Dans le roman, on constate également la volonté de Mathilde de réprimer à tout moment sa colère : «Elle veut le [Aristide Pommier ${ }^{56}$ ] voir. Elle lui dira qu'elle sait. Elle lui demandera pardon comme la fille bien élevée qu'elle est quand elle ne traite pas les gens de mange-merde. Il n'aura plus de scrupule à défendre, il lui parlera » $(L D F, 89)$. Cependant, dans ce cas, il s'agit plutôt d'une stratégie dont le but est d'obtenir des informations de l'ancien cuisinier

${ }^{55}$ Cf. Erving GOFFMAN, La mise en scène de la vie quotidienne, t. 2 Les Relations en public, Paris, Éditions de Minuit, coll. « Le Sens Commun », 1973.

${ }^{56}$ Militaire appartenant au même régime que Manech. 
du régiment, ce qui montrerait à nouveau sa sagacité policière. L'autre intérêt de la citation, c'est de révéler, par la voix de la narratrice, l'envie tranquille de Mathilde(r) de qualifier vulgairement Aristide Pommier. Cependant, sa finesse et son tact lui interdit cette franchise de comportement dans l'intérêt de sa quête.

Dans le film, l'étouffement des émotions de Mathilde(r) est complété par l'expressivité de sa physionomie, particulièrement de son regard. Celui-ci remplace la parole de Mathilde(f), et de la narratrice, dans plusieurs scènes avec le reste des personnages. Le regard sans cligner des yeux d'Audrey Tautou dans le rôle de Mathilde(f) révèle une palette d'émotions oscillant entre l'amour, la crainte, la déception, le mépris, l'agressivité ou l'optimisme. Qu'elle soit solitaire ou en compagnie, le public seul est témoin de l'expressivité de son regard fixe.

De très gros plans ou de gros plans focalisent les grands yeux noirs de Mathilde(f) exprimant sa sérénité, son acceptation, son appréhension, sa distraction, sa rêverie, son ennui, sa contrariété ou son intérêt. Ses yeux dotés d'un éclat brillant et saisissant révèlent également sa joie, sa confiance, sa peur, sa surprise, sa tristesse, son dégoût ou sa colère. Ses yeux fixes derrière ses paupières annoncent l'extase, son admiration, son chagrin ou sa rage. Lorsque la bouche de Mathilde(f) reste muette et ses lèvres fermées, c'est son regard qui l'exprime au-delà des scénarios et qui confie aux spectateurs ses réflexions et ses pulsions, ou bien comme l'assure Escartín « como barrera defensiva del mundo interior [...] frente al exterior $»^{57}$. Dans le roman Japrisot ne donne pas une telle importance au regard, la vue y est présent mais comme moyen de communication.

Constatons de même dans sa conversation avec les autres personnages le recours à expression corporelle. Citons, par exemple le commentaire de la narratrice lorsque Célestin Poux apparaît en pleine nuit :

\section{VOIX OFF}

Et Mathilde crispe ses mains l'une contre l'autre pour s'empêcher de trembler et de pleurer, d'être une bécasse qui fait honte...

Ses poings serrés, l'un contre l'autre, cachant sa bouche, l'empêchent de pleurer mais également d'exprimer sa surprise, sa joie et son espoir dans une quête qui semblait être au point mort.

\footnotetext{
${ }^{57}$ Montserrat ESCARTÍN GUAL, op. cit., p. 204.
} 

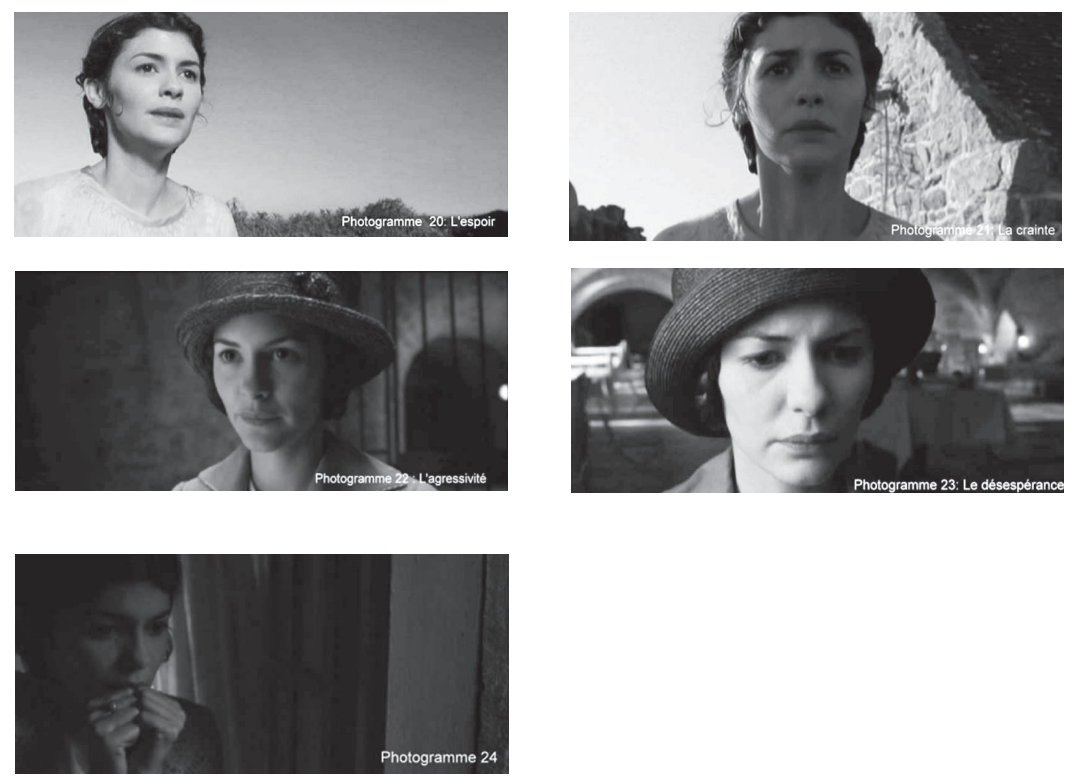

Un geste qui se répète nombreuses fois et semble révélateur de sa personnalité, c'est celui de ses mains croisées sur ses jambes jointes, par exemple face Daniel Espéranza (13:30), Germain Pire (29:26) ou Tina Lombardi (1:32:17). Ce geste traduirait soit une tension intérieure soit, selon Bruno Tiziana et Gregor Adamczyck, le souhait de «mettre un terme à la conversation $»^{58}$.

\section{En guise de non conclusion}

Dans les épigraphes précédentes, nous avons analysé et comparé la personnalité des héroïnes de Sébastien Japrisot et de Jean-Pierre Jeunet et Guillaume Laurant. Mathilde(r) et Mathilde(f) partagent la même histoire, mais appartiennent à des discours différents. Dans le processus d'adaptation, l'intrigue et les séquences du roman sont conservées, modifiées ou supprimées au bénéfice d'un nouveau récit qui accentue la présence de Mathilde(f) dans l'histoire.

\footnotetext{
${ }^{58}$ Bruno TIZIANA et Gregor ADAMCZYCK, Comprendre le langage du corps. Et percer les secrets de la communication non verbale, Ixelles, Ixelles éditions, 2011, p. 90.
} 
L'apparition de Mathilde(f) sur l'écran est extrêmement soignée du point de vue esthétique, ainsi rien n'est laissé au hasard par les costumières, les maquilleurs ou les coiffeurs.

Nous avons particulièrement pu constater certains aspects très significatifs des coiffures de Mathilde(f) avant, durant et après la disparition de Manech. Normalement, en public elle apparait avec un chapeau cloche ou un canotier pour dame. Nous sommes conscients qu'à l'époque, les chapeaux étaient en vogue, mais en faisant intervenir de nouveau la symbolique, le chapeau représente l'autorité, le pouvoir $^{59}$, deux qualités qui définissent Mathilde(f) dès le début de l'histoire.

En privé et en public durant l'absence de Manech, la coiffure de la jeune femme est constituée de deux petits chignons de chaque côté de la tête, entourés par des tresses qui cachent ses oreilles. Cette coiffure, rappelant les portraits de femmes de Léonard de Vinci, par exemple La Belle Ferronière (1495- 1499), recouvre presque la moitié de son visage et assombrit son aspect. Par contre, durant les temps heureux, puis à nouveau lors de sa rencontre avec Manech, ses cheveux doux et souples sont tirés en arrière sur la nuque et réunis en un chignon, qui laisse voir son visage resplendissant.

Ce détail concernant uniquement la coiffure, pourrait être étendue aux vêtements ou au maquillage, cette étude sur la personnalité de Mathilde(f) pourrait ainsi être étendue et complétée par la prise en compte de ces éléments qui nous révèleraient de nouveaux traits de sa personnalité face à l'autre Mathilde(r), personnage de papier et d'encre qui ne prend forme que dans l'imaginaire de chaque lectrice, de chaque lecteur.
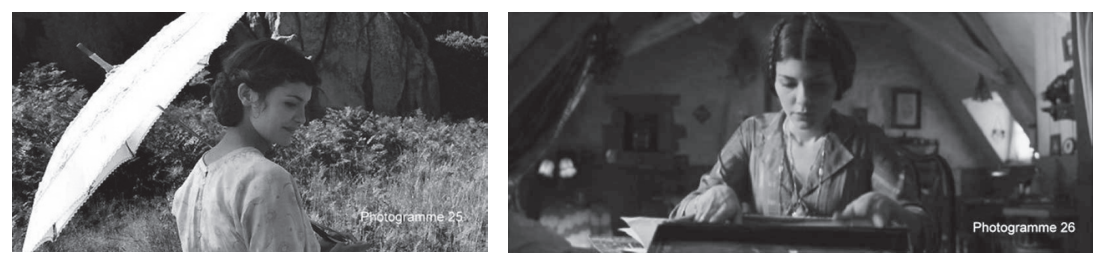

${ }^{59}$ Cf. J.C. COOPER, Diccionario de símbolos, México, Gustavo Gili, 2000, p. 170. 


\section{Références bibliographiques}

ARTIAGA, Loïc, Le roman populaire : des premiers feuilletons aux adaptions télévisuelles, 1836-1960, Paris, Autrement, 2008.

AUDOIN-ROUZEAU, Stéphane et Annette Becker, 14-18, retrouver la Guerre, Paris, Gallimard, 2000.

BAUDORRE, Philippe, « Pour l'exemple », in Richard JACQUEMOND (dir), Histoire et fiction dans les littératures modernes. (France, Europe, monde arabe). L'écriture de l'histoire. Volume 2, Paris, L'Harmattan, 2006, pp. 29-44.

BLETON, Paul, «Les fortunes médiatiques du roman populaire », in Loïc Artiaga (dir), Le roman populaire: des premiers feuilletons aux adaptations télévisuelles, 1836-1960, Paris, Éd. Autrement, 2008, pp. 137- 155.

BRAYET, Aurélie, Revivre : victimes de guerre de la Grande guerre à SaintÉtienne 1914-1935, Saint-Étienne, Publications de l'Université SaintÉtienne, 2006.

CATTEL, Raymond B., Personality : A systematic theoretical and factual study, New York, McGraw-Hill, 1950. Cité par Huber WINFRID, Introduction à la psychologie de la personnalité, Liège, Mardaga, 1977, p. 12.

CHAUMIER, Serge, L'amour fissionnel : Le nouvel art d'aimer, Paris, Fayard, 2004.

CHEVALIER, Jean et Alain Gheerbrant, Dictionnaire des symboles, Paris, Robert Laffont, 1982.

COCHET, François, lère Guerre mondiale : date, thèmes, noms, Paris, Studyrama, 2001.

COHEN, David, Comment décoder les gestes de vos interlocuteurs, Paris, LEDUC, coll. « DEVELOPPEMENT », 2010.

COOPER, J.C., Diccionario de símbolos, México, Gustavo Gili, 2000.

DENEB, Léon, Diccionario de símbolos : selección temática de los símbolos más universales, Madrid, Biblioteca nueva, 2000.

ESCARTÍN GUAL, Montserrat, Diccionario de símbolos literarios, Barcelona, PPU, 1996.

FREUD, Sigmund, Cinq leçons sur la psychanalyse, Paris, Payot, 1966.

GOFFMAN, Erving, La mise en scène de la vie quotidienne, t. 2 Les Relations en public, Paris, Éditions de Minuit, coll. « Le Sens Commun », 1973.

HUBER, Winfrid, Introduction à la psychologie de la personnalité, Liège, Mardaga, 1992.

JAPRISOT, Sébastien, Un long dimanche de fiançailles, Paris, Denoël, 1991. Un long dimanche de fiançailles, Paris, Folioplus, coll. « Classiques du $\mathrm{XX}^{\mathrm{e}}$ siècle », Paris, 2004. 
JUNG, C.G., L'Homme et ses symboles, Paris, Robert Laffont, 1964.

KÖRÖMI, Gabriella, "Narration d'une double recherche dans Un long dimanche de fiançailles de Sébastien Japrisot », Verbum Analecta Neolatina, XII/2, pp. 329-345.

LOUBET-POËTTE, Vanessa, L'énonciation cinématographique. Caractéristiques et méthode(s) d'analyse d'une énonciation artistique audio-visuelle dans les longs métrages de Jean-Pierre Jeunet. Delicatessen, La Cité des enfants perdus, Alien Résurrection, Le Fabuleux destin d'Amélie Poulain, Un long dimanche de fiançailles, Tome 1. Thèse pour le Doctorat en Linguistique française. Sous la direction de M. le Professeur Jean-Gérard LAPACHERIE, 2010.

MENARD-LEMEUNIER, Louise, « Jour de Toussaint », in Le trait d'union, novembre 1924, Archives Départementales de Maine-et-Loire, 126 JO 1.

MERCIER, Michel, L'identité handicapée, Namur, Presses Universitaires de Namur, 2004.

MILLET, Kate, La Politique du mâle, Paris, Stock, coll. « Points actuels », 1971.

MOUNIN, Georges, Introduction à la sémiologie, Paris, Minuit, 1970.

NECKER, Germain de, Corinne ou l'Italie, Paris, Gallimard, coll. « Folio Classique », 1992.

PERVIN, Lawrence A. et Oliver P. John, La personnalité : de la théorie à la recherche, Québec, Éditions du Renouveau Pédagogique INC, 2005.

PETIT, Stéphanie, Les veuves de la Grande Guerre, d'éternelles endeuillées ?, Paris, Cygne, 2007.

ROVENTA-FRUMUSANI, Daniela, Concepts fondamentaux pour les études de genre, Paris, Éditions des archives contemporains, coll. « Archives des études de genre », 2009.

SAND, George, Teverino, Babel n 437, Arles, Actes Sud, 2003.

SCHOENTJES, Pierre (éd.), La Grande Guerre : Un siècle de fictions romanesques, Genève, Librairie Droz, 2008.

TIZIANA, Bruno et Gregor Adamczyck, Comprendre le langage du corps. Et percer les secrets de la communication non verbale, Ixelles, Ixelles éditions, 2011.

TODOROV, Tzevetan, Théories du symbole, Paris, Seuil, 1977.

VATRE, Françoise et Vincent Fries, «Les oubliés de l'amour », in Michel Mercier, L'identité handicapée, Namur, Presses Universitaires de Namur, 2004, pp. 75-86. 


\section{Sitographie :}

http ://www.latribunerepublicaine.fr/Actualite/Bellegarde/2011/08/24/article delphine_arene_la_dame_duhaut_bugey.shtml. [page consultée le 5 novembre 2014]

http ://www.allocine.fr/critique/fichepresse_gen_cpresse=82001.html?page $=5$. [page consultée le 20 octobre 2014] 